\title{
DEVELOPMENT OF A HUMIDITY-RESISTANT COATING TO IMPART HIGH OXYGEN BARRIER PERFORMANCE TO FOOD PACKAGING FILMS
}

\author{
A Thesis \\ presented to \\ the Faculty of California Polytechnic State University, \\ San Luis Obispo
}

\author{
In Partial Fulfillment \\ of the Requirements for the Degree \\ Master of Science in Polymers and Coatings
}

by

Ryan Yinghua Cox

June 2017 
(C) 2017

Ryan Yinghua Cox

ALL RIGHTS RESERVED 
TITLE:

AUTHOR:

DATE SUBMITTED:

COMMITTEE CHAIR:

COMMITTEE MEMBER:

COMMITTEE MEMBER:
Development of a Humidity-Resistant Coating to Impart High Oxygen Barrier Performance to Food Packaging Films

Ryan Yinghua Cox

June 2017

Raymond Fernando, Ph.D.

Professor of Polymers and Coatings Science

Director of Polymers and Coatings Program

Department of Chemistry and Biochemistry

Ajay Kathuria, Ph.D.

Assistant Professor of Business

Department of Industrial Packaging and

Technology

Ben Caes, Ph.D.

Innovation Manager CUSA

Siegwerk Corporation 


\begin{abstract}
Development of a Humidity-Resistant Coating to Impart High Oxygen Barrier Performance to Food Packaging Films

\section{Ryan Yinghua Cox}

Oxygen barrier coatings have the potential to greatly extend the lifetime of certain food products by incorporating them into existing food packaging. Present technologies face definite challenges of maintaining high performance, while attaining simple and inexpensive preparation methods. The oxygen barrier effect obtained with these coatings is also susceptible to a plasticization effect when exposed to high humidity, since water vapor molecules are readily soluble in typically hydrophilic resins. In this work, we demonstrate a $1-2$ micron thick oxygen barrier coating, prepared on a 12 micron poly(ethylene terephthalate) substrate, that has oxygen transmission rates as low as 1.44 $\mathrm{cc} \mathrm{m}^{-2}$ day $^{-1}$ under standard conditions and can maintain similar oxygen barrier performance at high humidity. This degree of oxygen barrier meets the standard of $1-10$ $\mathrm{cc} \mathrm{m}^{-2}$ day $^{-1}$ established for food packaging applications. The coating is prepared through use of sol-gel chemistry between poly(vinyl alcohol) and vinyltrimethoxsilane molecules, which form a strong network resin through hydrolysis and condensation reactions. The formulation of these oxygen barrier coatings allows for variability of solids percentage and viscosity without significant change in performance. The ability to scale up the preparation of these coated films was tested successfully on an industrial flexographic printing press. 


\section{ACKNOWLEDGMENTS}

First and foremost, I would like to thank my advisor, Dr. Ray Fernando, for approaching me with the opportunity to join this project. Over the last year, he has provided insight and assistance, while generally allowing me to take strong ownership of this research to the degree that would be expected in industry.

I would like to thank Dr. Ben Caes and Dr. Scot Pedersen from Siegwerk Corporation for providing the chance to turn their initial concept into the product described herein. Their advice and direction helped drive progress during the project's duration.

I would like to thank two professors, Dr. Ajay Kathuria and Dr. Malcolm Keif, who have both selflessly taken extensive time out of their schedules to help make this project a success. In their respective fields, each of these professors have helped me understand the complex background information related to preparation and testing of these films. Dr. Kathuria's involvement allowed use of the MOCON OX-TRAN instrument in the Industrial Packaging and Technology department. Dr. Keif allowed us to utilize several important film preparation techniques, including use of the QD Proofer and the Mark Andy flexographic printing press located within the Graphic Communications department. I am grateful to both of these departments for allowing use of facilities and their general support on the project.

I would like to express extensive thanks to Travis Smith, Research Associate, for providing priceless thoughts and assistance week after week. Without him by my side for all stages of the project, I don't think I could have achieved what we accomplished developing this oxygen barrier coating, overcoming all challenges that we faced. 
I would like to thank Siegwerk Corporation and the Bill Moore Endowment for Financial Support for providing funding for the project. This financial support aided collaboration between departments, and provided the materials that were essential to putting the project into action.

I would like to thank my colleagues Josh Wolcott, Jake Asola, and Jenny Liang for their assistance during the production stages of the project. These undergraduate students worked around their schedules, and took great care in the film preparation and oxygen transmission rate testing to help achieve the goals of the project.

Thank you to the Chemistry and Biochemistry department for permission to use the Kenneth N. Edwards Western Coatings Technology Center for this project. Additional thanks must be expressed to this department for providing an excellent education, caring professors, research opportunities, chemicals, funding, and support throughout my years at California Polytechnic State University, San Luis Obispo.

Finally, I would like to thank all of my friends, family, professors, advisors, and fellow students for always being there to support my progression towards becoming a scientist focused in the coatings industry and helping to achieve the success that I have been lucky enough to be a part of during my time as a student. 


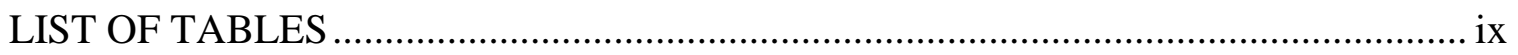

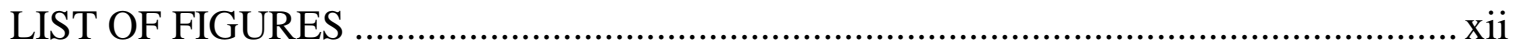

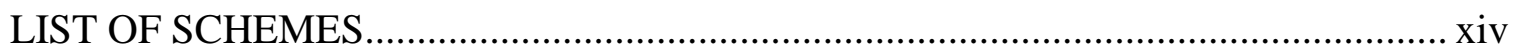

\section{CHAPTER}

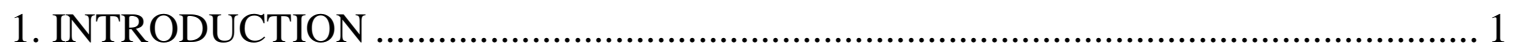

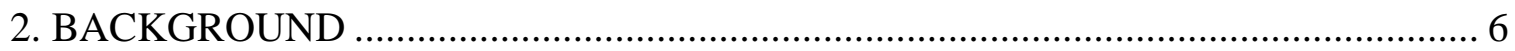

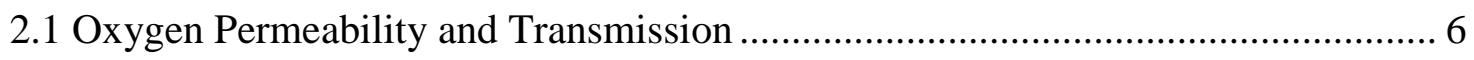

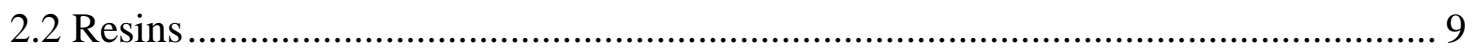

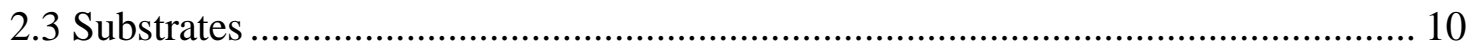

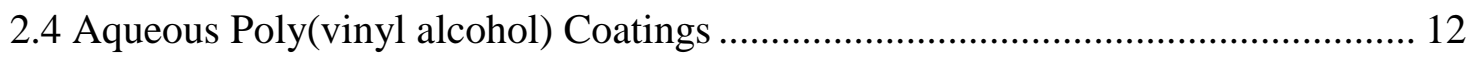

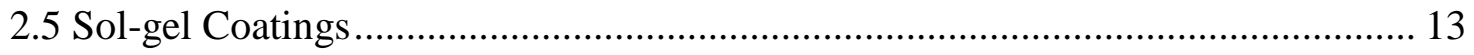

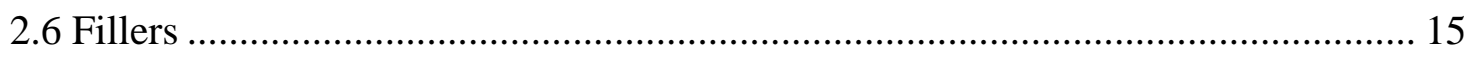

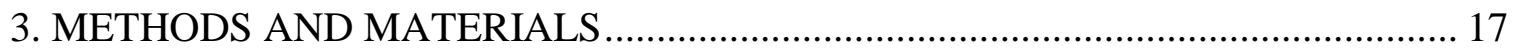

3.1 Materials Used During Coating Preparation........................................................ 17

3.2 Preparation of Over-Print Varnish Polyurethane Coatings with Added Fillers...... 18

3.3 Preparation of Sol-Gel Coatings ……............................................................ 18

3.4 Preparation of Poly(vinyl alchohol) \& Vinyltrimethoxysilane Coatings ............... 19

3.5 Preparation of Coated Films Using the QD Proofer ............................................. 20

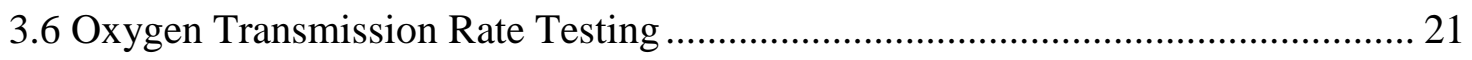


4. RESULTS AND DISCUSSION

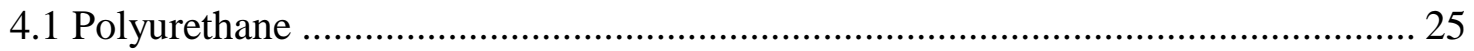

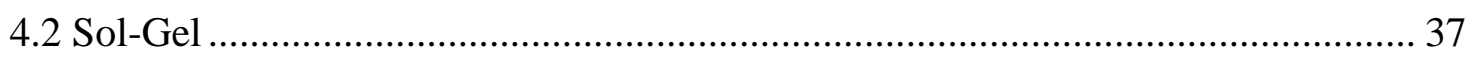

4.3 Poly(vinyl alchohol) \& Vinyltrimethoxysilane ............................................... 46

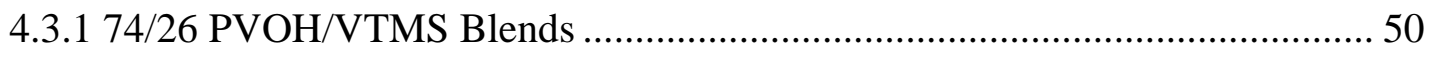

4.3.2 85/15 and 97/3 PVOH/VTMS Blends ................................................... 55

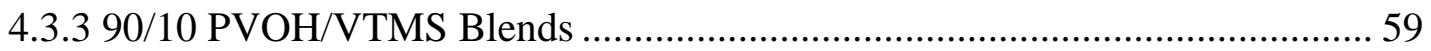

4.3.4 Diluted 90/10 PVOH/VTMS Blends ...................................................... 61

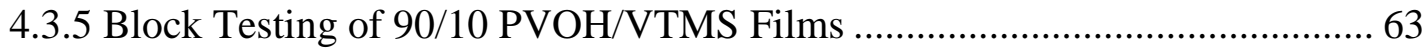

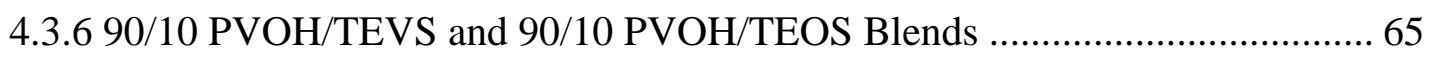

4.3.7 90/10 PVOH/VTMS Blend Prepared without Heat....................................... 67

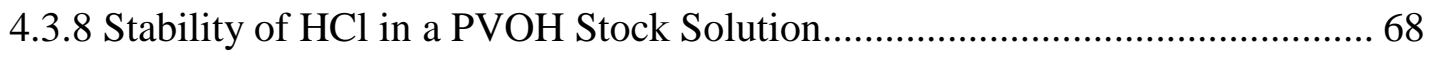

4.3.9 90/10 PVOH/VTMS Blend Prepared with Citric Acid.................................. 69

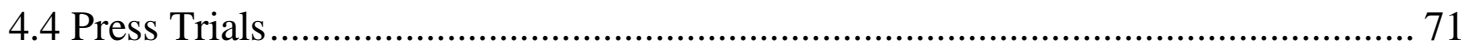

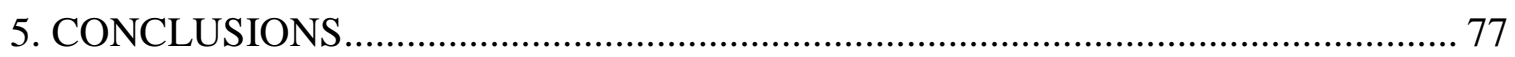

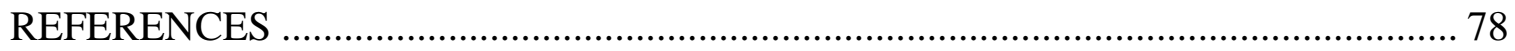




\section{LIST OF TABLES}

Page

Table 1. OTR comparison of common polymer films used as substrates in the food

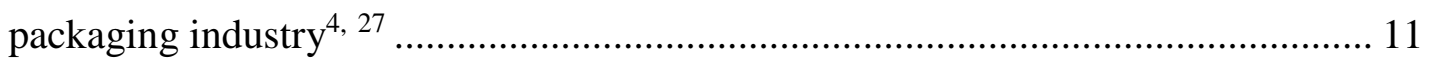

Table 2. 35\% NVW OPV polyurethane formulation with and without added filler ........ 27

Table 3. Description of filler material compositions, median particle diameters, and

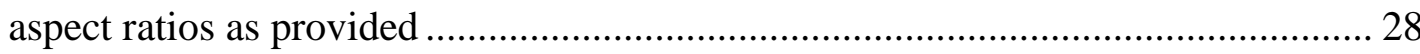

Table 4. Summary of sonication times necessary to achieve proper filler dispersion and coating quality for each filler material 30

Table 5. Oxygen transmission rates and related thicknesses for the $30 \%$ NVW PU formulations 34

Table 6. 50\% NVW OPV PU formulation with and without filler. 36

Table 7. Oxygen transmission rates and thicknesses obtained for the 50\% NVW PU formulations 37

Table 8. Sol-Gel formulation utilizing APTS and Epon 1001-X-75 ............................. 40

Table 9. Sol-Gel formulation utilizing APTS and ERL 4221 ................................... 41

Table 10. Sol-gel formulation utilizing TEOS, APTS, and PTOL ............................... 43

Table 11. Sol-gel formulation utilizing TEOS, GPTMOS, and ERL 4221 .................... 44

Table 12. Oxygen transmission rate and properties for the sol-gel systems................... 45

Table 13. Summary of reagent quantities and resultant properties for the 74/26 PVOH/VTMS blends 51

Table 14. Oxygen transmission rates and thicknesses obtained for the $\mathrm{PVOH}$ and 74/26 PVOH/VTMS films 
Table 15 . Summary of reagent quantities and resultant properties for the $85 / 15$ and 93/7 PVOH/VTMS blends 56

Table 16. Oxygen transmission rates and thicknesses obtained for the 85/15, 93/7 single layer, and 93/7 double-layer PVOH/VTMS films. 57

Table 17. Summary of properties and reagent quantities for the 90/10 PVOH/VTMS

blend. 60

Table 18. Oxygen transmission rates and thicknesses obtained for the 90/10

PVOH/VTMS films 60

Table 19. Summary of properties and reagent quantities for the diluted 90/10

PVOH/VTMS blends

Table 20. Oxygen transmission rates and thicknesses obtained for the diluted 90/10 PVOH/VTMS films

Table 21. Summary of properties and reagent quantities for the 90/10 PVOH/TEVS and 90/10 PVOH/TEOS blends 65

Table 22. Oxygen transmission rates and thicknesses obtained for the 90/10 PVOH/TEVS and 90/10 PVOH/TEOS films 67

Table 23. Summary of properties and reagent quantities for the 90/10 PVOH/VTMS blend prepared with a citric acid catalyst 70

Table 24. Oxygen transmission rates and thicknesses obtained for the 90/10

PVOH/VTMS films prepared with a citric acid catalyst 71

Table 25. Summary of properties and reagent quantities for the 90/10 PVOH/VTMS blend used for scaled up press run 72 
Table 26. Oxygen transmission rates and thicknesses obtained for the 2 mil PET press trial films using the 90/10 PVOH/VTMS blend............................................. 76 


\section{LIST OF FIGURES}

Page

Figure 1. Illustration of the basic concept of oxygen barrier coatings............................ 3

Figure 2. Chemical structures of a) PVOH and b) EVOH repeat units .......................... 12

Figure 3. Illustration of the different outcomes of filler integration within an existing

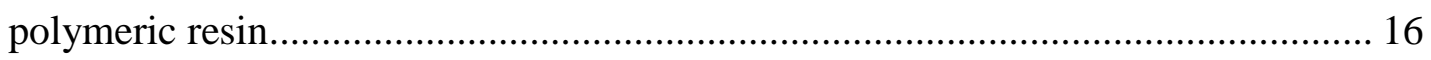

Figure 4. POM image of unfilled (left) and 1 wt.\% Stellar 420 (right) OPV 50\%

NVW PU films at 5x magnification........................................................ 33

Figure 5. Chemical structures of several commonly used alkoxysilane molecules.......... 38

Figure 6. POM image of the TEOS/GPTMOS/ERL 4221 films at 5x magnification ...... 46

Figure 7. ATR-FTIR overlay of the traces of VTMS (purple), PVOH (green), and

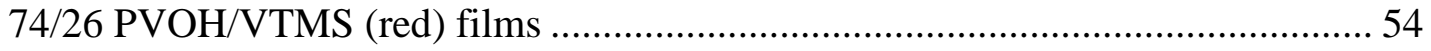

Figure 8. POM image of the 74/26 PVOH/VTMS film (left) compared to an

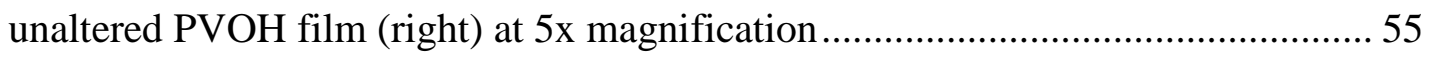

Figure 9. POM images of the 85/15 (left) and 74/26 (right) PVOH films at 5x

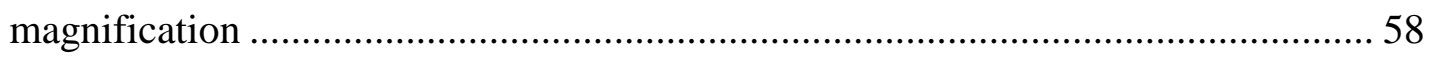

Figure 10. POM images of the 90/10 PVOH/TEVS film (left) and 90/10

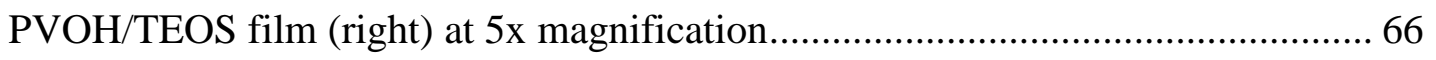

Figure 11. ${ }^{1} \mathrm{H}$ NMR overlay of traces for unreacted PVOH (blue) and the $\mathrm{PVOH} / \mathrm{HCl}$ mixture (crimson)

Figure 12. Images of the up scaled, round bottom reaction setup (left) and Mark Andy flexographic press (right). 73 
Figure 13. POM images of M504 2 BCM (top left), M541 2 BCM (top right), M504 6 BCM (bottom left), and M541 6 BCM (bottom right) films at 5x magnification with double-layers above and single-layers below each diagonal line. 


\section{LIST OF SCHEMES}

Page

Scheme 1. The two-step reaction mechanism found in sol-gel chemistry involving a) hydrolysis of the alkoxysilane and b) condensation of multiple hydrolyzed silane molecules 14

Scheme 2. The condensation reaction that would occur between a mixture of PVOH and an alkoxysilane molecule, from which additional reactions could take place to form a network structure

Scheme 3. Typical reaction mechanism between a diisocyanate molecule and a polyol forming a polyurethane. 26 


\section{INTRODUCTION}

The development of improved food packaging technologies is driven by the extent of food waste occurring worldwide. Traditionally, the food supply chain is divided into several distinct stages: field growth, post-harvest, processing, distribution, retail, and consumer use. ${ }^{1}$ The latter two stages mentioned are where enhanced food packaging could provide the largest impact. Packaged foods are typically kept under conditions during the retail and consumer stages that can be detrimental to the quality of the food. This is due to limitations on how well environmental aspects can be controlled while keeping the product available and attractive to the costumer. Food packaging can be modified to provide varying amounts of control over most of these conditions, often resulting in better food preservation.

Looking at recent statistics, food waste continues to grow over time as global populations simultaneously increase. In 1995, the mass of food lost to waste by stores and households was estimated at 8.6 million metric tons, accounting for roughly half of the loss over the entire supply chain. ${ }^{2}$ Studies from 2011 have reported that 1.3 billion metric tons of food is wasted throughout all stages of the supply chain, which accounts for a third of all food produced worldwide. ${ }^{3}$ This inefficiency within the food supply chain is likely to have a major impact on society if they continue to increase with time. An important distinction between food waste occurring in developed countries, like Europe and North America, compared to developing countries, such as Africa and Latin America, is the percentage of waste caused by the consumer. In developed countries, this percentage is on average $30-40 \%$, while consumer waste only accounts for $5-15 \%$ in developing countries. ${ }^{3}$ This contrast highlights a societal difference in developed 
countries, where food is seen as less of a luxury item, leading to increased consumer waste.

Progress in food packaging technology promises an increase in food stability and lifetime that could reduce losses found at both the retail and consumer levels. Higher reduction would then be expected for developed countries due to the consumer mentality towards food use, and a greater capability to invent and integrate new food packaging into existing food packaging systems.

Variables that affect food degradation can be separated into two main classifications: intrinsic factors and extrinsic factors. Intrinsic factors include $\mathrm{pH}$, microbiological activity, chemistry of the product, and the possible interactions between the packaging material and the food product. ${ }^{4}$ These factors are uncontrollable to a certain extent since they are dependent on the composition of the food involved. Extrinsic factors are defined as the environment that the food product will experience during its shelf life and consumer usage, including processing conditions, heat exposure and regulation, relative humidity, ultraviolet (UV) and infrared (IR) radiation exposure, and the internal atmospheric composition.

Oxygen barrier coatings, the focus of this project, can control the internal gas composition within the food packaging by limiting the degree to which oxygen gas permeates through the packaging that surrounds the perishable food. The main food types which are prone to oxygen degradation routes are red meats, fish, and nuts. Oxygen concentrations as low as 1-200 parts per million have been determined to lead to major loss in food quality. ${ }^{5}$ The higher amounts of unsaturated lipids in these oily, fatty foods makes them highly susceptible to oxidation by a variety of reactive oxygen derivatives, 
including oxide radicals, hydrogen peroxide, and hydroxyl radicals. These chemical species are produced in low percentages, $5-10 \%$, during respiration. ${ }^{6}$ Peroxidation of these polyunsaturated fatty acids form a range of unwanted products that negatively affect the taste and quality of the food. Red meats, in particular, also suffer from significant browning due to oxygen radical reactions with myoglobin. ${ }^{7}$ Regular myoglobin is converted into metmyoglobin, which in high concentrations changes the food's appearance. While appearance may not seem as important as food quaily, an improvement of one to two days in color retention would save an estimated \$175 million to $\$ 1$ billion annually for the US meat industry. ${ }^{7}$

Prevention of this undesired oxidation degradation has traditionally been achieved through storage in dry conditions, which can slow the kinetics of lipid oxidation, and by the introduction of oxygen scavengers or pre-determined atmospheric compositions to the internal environment. ${ }^{8}$ Recently, oxygen barrier coatings have emerged as a highlycontrollable component of food packaging, that can help reduce oxygen permeation and control the atmosphere surrounding the food product (Figure 1).
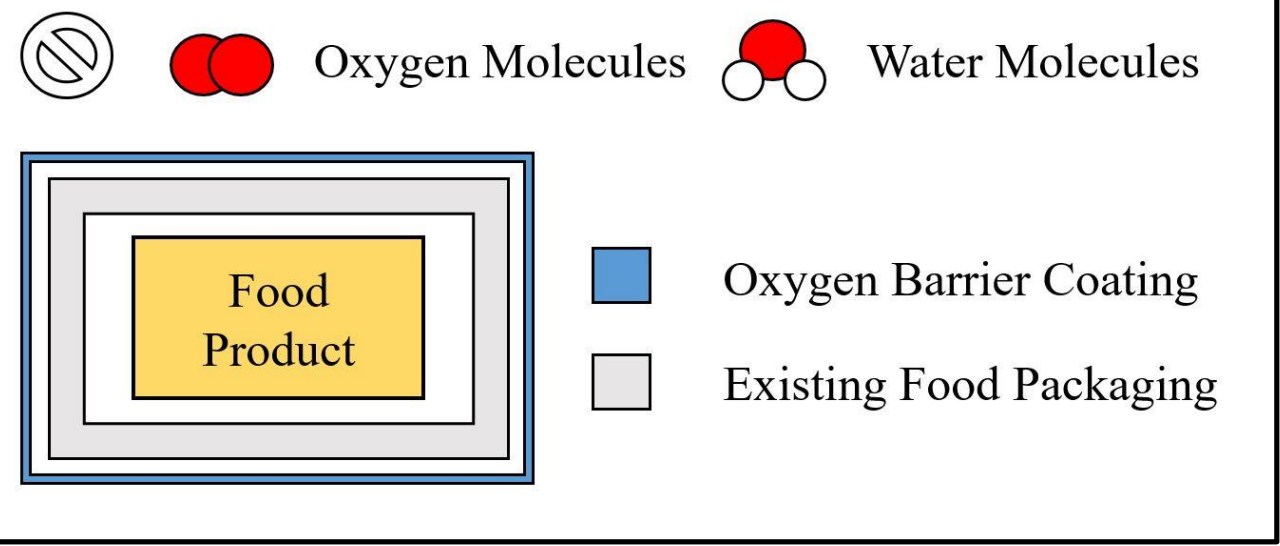

Figure 1. Illustration of the basic concept of oxygen barrier coatings 
Prominent integration of oxygen barrier plastics and coatings can be traced back to the 1980s. Initial oxygen barrier coatings consisted of metalized plastics that were created using vacuum deposition methods. This technique added a layer of metal, typically aluminum, onto existing plastic films to improve thermal, barrier, and mechanical properties. ${ }^{9}$ A similar approach involved a multilayer design, wherein several thin layers of varying composition were used, whose overall thickness was the same as a single layer of coating. ${ }^{10}$ A large number of patents, granted during the 1980s, show the quick development of oxygen barrier technologies from metalized films ${ }^{11}$ to enhanced solventbased barrier coatings. ${ }^{12-14}$ These older oxygen barrier coating systems gave a strong foundation upon which future technologies could be developed.

Current day oxygen barrier coatings closely resemble those from thirty to forty years ago, yet bring in new deposition methods or resin preparation routes that provide high reduction in oxygen permeability of $70 \%$ or more. Review of recent literature highlights the variety of compositions and techniques used to achieve this high barrier performance. The largest improvements typically result from expensive, high precision deposition methods that provide nano-thin films such as atomic layer deposition (ALD) $)^{15}$, chemical vapor deposition $(\mathrm{CVD})^{16}$, and plasma-enhanced chemical vapor deposition (PECVD) ${ }^{17}$ Several studies have also demonstrated complicated resin structures that can provide strong oxygen barrier performance, such as perhydropolysilazanes ${ }^{18}$ and polyhedral oilgosilsesquioxanes. ${ }^{19}$ Complex variations of oxygen barrier coatings have been created with the ability to self-heal. ${ }^{20}$ In order to be viable from an industrial standpoint in terms of complexity and cost, simpler compositions and deposition routes are preferred. One approach utilizes the combination of an intrinsically strong resin with an inorganic platy 
filler material. Systems using polyurethanes with montmorillonite tactoids and Lihectorites $^{21}$ additions and polyetheylenimine with montmorillonite clay ${ }^{22}$ are common examples of this composition. Inorganic-organic polymers, typically produced through a sol-gel mechanism, can provide another promising oxygen barrier resin. ${ }^{5}$ The next step in the evolution of food packaging will likely be due to an oxygen barrier coating that can be readily and harmlessly integrated into current industrial processing practices. 


\section{BACKGROUND}

\subsection{Oxygen Permeability and Transmission}

To better understand oxygen barrier performance, a review of oxygen permeation fundamentals is required. The permeability of oxygen $(\mathrm{P})$ depends on two parameters, the solubility coefficient (S) and diffusivity coefficient (D) as given by Equation 1.

$\boldsymbol{P}=\boldsymbol{S} * \boldsymbol{D}$

(Equation 1)

To model the permeation of an oxygen molecule through a coating, there are two main transitions that must be considered. First, the oxygen molecule must absorb into the polymeric coating, a process dictated by oxygen's solubility in the particular polymeric resin. This transition must also take place as the oxygen molecule desorbs from the coating. Generally, solubility of a gas within a solid or liquid is generally affected by polarity. Introduction of a polar resin lowers the solubility of oxygen molecules within the coating; called a solubility barrier effect.

Next, the oxygen molecule must then diffuse through the depth of the coating, a process dictated by the diffusivity of the oxygen molecule and the coating thickness. In the simplest case, the oxygen molecule is able to freely travel in a direct path across the coating layer without any change in the diffusion rate; as in an amorphous polymer. The introduction of ordered internal structure like crystallinity or micro-sized domains can effectively block the oxygen molecule from taking a direct path through the coating. Whether achieved from intrinsic crystallinity or the introduction of platy filler materials, oxygen molecules are highly insoluble in these domains and are forced to take a longer path around the affected areas. As a result of the longer distance that the oxygen molecules take to travel through the coating, the oxygen permeation is lowered. This 
concept is often referred to as the 'more torturous path' in literature, and will be referred to as a diffusivity barrier effect in this study. Diffusivity barrier effects are typically the main mechanism through which strong oxygen barrier coatings control oxygen permeation.

There are several other factors that can also influence oxygen permeation, such as temperature, humidity, and defects. Temperature always plays an important role whenever kinetics are involved, and higher temperatures have been shown to result in significantly raised oxygen transmission rates. ${ }^{23}$ Typically, oxygen barrier films are tested at high humidity to see if performance is withheld, which will be discussed in more detail as a significant challenge for this project. Many oxygen barrier coatings degrade in the presence of water, as these polar molecules are readily soluble in their resin structure and can break it apart. The presence of defects within the coating sample facilitate oxygen diffusion causing a diminished barrier performance. Models suggest that defects with a certain radius in the horizontal plane will dominate the diffusion rate in a coating depth three times that of the defect radius. ${ }^{24}$ Since a small quantity of defects can completely ruin the performance of a barrier coating, the use of sequential layering and optimization of the coating deposition are vital to ensure the success of an oxygen barrier coating.

The movement of oxygen through a coated film is typically referred to in terms of oxygen transfer, rather than oxygen permeability, since transfer rate is the quantity that is directly measured. This quantity is known as the oxygen transmission rate (OTR) and is defined as the volume of permeant gas (cubic centimeters or cc) that travels through a constant film area (square meters or $\mathrm{m}^{2}$ ) over a specified time frame (day). The difference between OTR and oxygen permeability, is that the thickness of the film $(\mu \mathrm{m})$ and the 
pressure differential (atmospheres or atm) are not accounted for in the OTR expression, as shown in Equations 2 and 3.

$$
\begin{aligned}
& \text { OTR }=\frac{\text { volume of permeant gas }}{(\text { film area }) *(\text { time duration })}=\frac{c c}{m^{2} \times \text { day }} \\
& P=\frac{(\text { volume of permeant gas }) *(\text { thickness of film })}{(\text { film area }) *(\text { time duration }) *(\text { pressure differential })}=\frac{c c \times \mu m}{m^{2} \times \text { day } \times \text { atm }}
\end{aligned}
$$

If these two parameters are provided along with the oxygen transmission rate, the equivalent permeability can be readily calculated. Due to the dependence of OTR on thickness and pressure differential, this means that OTRs are only comparable if they are measured at the same film thickness and with the same pressure on either side of the film samples. The terms of oxygen transmission rate and permeability are often used synonymously in industry and literature.

Oxygen transmission rates are commonly measured following the method described in ASTM D3985. The OX-TRAN instrument series, produced by MOCON, provide versatile oxygen transmission rate instruments that comply with ASTM D3985. Several models for testing different types of films and containers are available. Effectively, a specific film area is sealed within a horizontal testing cell that is loaded into the OXTRAN instrument; usually done in duplicate. Each individual cell is filled with oxygen gas on the top side of the film, while a constant stream of a carrier gas is streamed across the other side. This carrier gas is used to carry any oxygen molecules that have permeated through the film into a coulometric detector. The influx of oxygen molecules passing through this sensor produces a distinct current proportional to the number of molecules. ${ }^{25}$ The pressure on either side of the coating is maintained at or near atmospheric pressure. An oxygen transmission rate that is considered low for food packaging applications is 
around $10 \mathrm{cc} \mathrm{m}^{-2} \mathrm{day}^{-1}$ under standard temperature and pressure conditions (STP) and 1 $\mathrm{cc} \mathrm{m}^{-2}$ day $^{-1}$ would indicate exceptional oxygen barrier performance. ${ }^{26}$ This value is important to keep in mind when evaluating the results and progress throughout the project.

\subsection{Resins}

The major component of all oxygen barrier coatings is the polymeric resin structure which can be modified to specific needs through specialized polymerization routes. The specific requirements necessary for oxygen barrier performance are well defined in literature and include a high degree of polarity, high chain stiffness, inertness to oxygen, high chain packing, intermolecular forces between chains, and high glass transition temperature. ${ }^{27-30}$ Most of these characteristics affect either solubility or diffusivity barrier effects. As discussed earlier, solubility barrier effect is due to the difference in polarity between the resin and the permeant gas. Oxygen gas is nonpolar, meaning that, to efficiently keep oxygen molecules from permeating through a coating, a highly polar resin structure should be chosen to lower the solubility of oxygen gas molecules within it. Diffusivity barrier effects result from a combination of contributions including tacticity of the polymer, packing or free volume of adjacent polymer chains, and the intermolecular forces possible between functional groups. The presence of strong intermolecular forces, like hydrogen bonding and strong dipole moments, can promote the formation of crystalline lamella structures due to the attraction caused between chains. Again, the structural effects that cause a barrier due to diffusivity are more imperative to the oxygen barrier performance than those that influence the gas' solubility. 
The oxygen permeability related to specific functional groups has been extensively studied, which reinforces the analysis done for the common polymeric substrates. The functional groups with the lowest permeability were alcohol/hydroxyl groups (-OH) and acrylonitrile groups (-CN). Both of these chemical structures provide solubility barrier due to their polar nature, and can hinder oxygen diffusion because of strong hydrogen bonding capability that tightens molecular arrangement or can influence crystalline structure. Comparatively, simple hydrocarbon $(\mathrm{C}-\mathrm{H})$ linkages provide insignificant solubility and diffusivity barrier effects, as they are nonpolar and can only form weak London dispersion forces. These carbon-hydrogen bonds are typically highly flexible as well, resulting in a high free volume. The difference in oxygen permeability between high barrier functional groups and these simple hydrocarbon bonds is three orders of magnitude. ${ }^{27}$ By analyzing the chemical structure, one can predict whether a given resin structure will provide significant barrier performance.

\subsection{Substrates}

While the barrier properties of the substrate are often negligible compared to that of the oxygen barrier coatings that reside on top of them, the substrate is still an important variable when considering the overall food packaging. The selection of substrate can influence the adhesion of the oxygen barrier coating. Even if an oxygen barrier coating generally provides excellent oxygen barrier performance, if the coating won't adhere well to a given substrate, then the barrier coating may not be viable for certain markets that utilize that substrate. Poor adhesion can lead to a wide range of defects within the coating, causing high oxygen permeation. Corona-treated polymeric films help solve adhesion problems by oxidizing the surface and raising surface energy. ${ }^{27}$ 
Over thirty different types of polymeric substrates have been used in some form for food packaging; polyolefins and polyesters are the most commonly used. ${ }^{28}$ Of those polymers, several show strong barrier performance. As shown in Table 1, the OTR values for 25 micron thick polymer films of a vinylidene chloride-vinyl chloride copolymer and ethylenevinyl alcohol (EVOH) copolymer have extremely low OTRs. These OTRs are mainly influenced by the polyvinylidene chloride (PVDC), commonly known as Saran wrap, and poly(vinyl alcohol) (PVOH) components of these copolymers. These high barrier polymers are semi-crystalline and contain polar functional groups, which limit oxygen diffusion and solubility, respectively. Poly(ethylene terephthalate) (PET) has moderate oxygen barrier properties and is commonly used to produce bottles and food packaging. Simple hydrocarbon polymers like polyethylene (PE) and polypropylene (PP) feature high OTRs on account of their simple internal structure and nonpolar nature.

Table 1. OTR comparison of common polymer films used as substrates in the food packaging industry ${ }^{4,27}$

\begin{tabular}{|c|c|}
\hline $\begin{array}{c}\text { Film Composition } \\
(\mathbf{2 5} \text { microns thick) }\end{array}$ & $\begin{array}{c}\text { OTR }\left(\mathbf{c c ~}^{\mathbf{- 2}} \mathbf{d a y}^{\mathbf{- 1}}\right) \\
\text { at 0\% RH }\end{array}$ \\
\hline Low-Density Polyethylene (LDPE) & $6500-8500$ \\
\hline High-Density Polyethylene (HDPE) & $1600-2000$ \\
\hline Cast Polypropylene & $3500-4500$ \\
\hline Poly(ethylene terephthalate) & $100-150$ \\
\hline Polyvinylidene Chloride & $2-4$ \\
\hline $\begin{array}{c}\text { Vinylidene Chloride - Vinyl } \\
\text { Chloride Copolymer }\end{array}$ & 1.25 \\
\hline Ethylenevinyl Alcohol & 0.2 \\
\hline Poly(vinyl alcohol) & $<0.1$ \\
\hline
\end{tabular}




\subsection{Aqueous Poly(vinyl alcohol) Coatings}

One of the strongest oxygen barrier polymers, poly(vinyl alcohol), deserves a mention (Figure 2). Both polymers provide OTRs that can be several orders of magnitudes lower than other packaging polymers. Since PVOH typically comes in the form of crystalline flakes, the easiest way to create a thin coating of this polymer is by utilizing its solubility in water at temperatures near PVOH's glass transition temperature

of $85^{\circ} \mathrm{C} .{ }^{31} \mathrm{By}$ approaching this temperature, an aqueous polymeric solution that is deposited through traditional liquid coating deposition methods, then dried and cured at elevated temperatures. EVOH, a copolymer of poly(vinyl alcohol) and polyethylene, provides comparable oxygen permeation to unaltered $\mathrm{PVOH}$ with significantly improved water resistance due to the nonpolar hydrocarbon segments introduced.
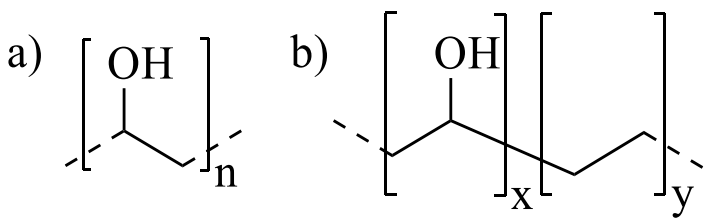

Figure 2. Chemical structures of a) $\mathrm{PVOH}$ and b) EVOH repeat units

Unlike most polymers, $\mathrm{PVOH}$ is synthesized through the hydrolysis of poly(vinyl acetate), making vinyl acetate $\left(\mathrm{CH}_{2} \mathrm{CHOCOCH}_{3}\right)$ the monomeric unit instead of vinyl alcohol $\left(\mathrm{CH}_{2} \mathrm{CHOH}\right)$. Hydrolysis of part of the acetate group under alkaline or acidic conditions provides poly(vinyl alcohol)'s structure. The degree of hydrolysis (in \%) has a direct effect on properties such as molecular weight, solubility, flexibility, tensile strength, and adhesiveness. ${ }^{32} \mathrm{PVOH}$ is most commonly used for coating applications with a degree of hydrolysis of $97-99 \%$. Due to the hydroxyl groups found regularly along each PVOH chain, there is strong inter-chain and intra-chain hydrogen bonding that 
promotes semi-crystallinity on the order of $20-30 \%$ which gives higher tensile strength compared to other polymers.

There are a few limitations that $\mathrm{PVOH}$ coatings have that can negatively affect its performance as an oxygen barrier coating. $\mathrm{PVOH}$ has a melting point of $215^{\circ} \mathrm{C}$ and tends to thermally degrade through dehydration at temperatures near its melting point. ${ }^{31}$ This makes the polymer unsuitable for melt processing and other high temperature processing techniques. The other major drawback of $\mathrm{PVOH}$ is that water molecules have high solubility in PVOH due to its highly polar nature. Water molecules effectively plasticize the PVOH resin causing defects to form within the coating. This is a common occurance for most high oxygen barrier coatings and other hydrophilic molecules like proteins and polysaccharides. ${ }^{33}$ At a relative humidity of $85-90 \%$, where water vapor concentration is high, this effect can reduce the oxygen barrier coatings to complete ineffectiveness.

\subsection{Sol-gel Coatings}

Sol-gel coatings prepared from alkoxysilanes $\left(\mathrm{HSiOR}_{3}\right.$ or $\left.\mathrm{SiOR}_{4}\right)$ represent another approach to preparing oxygen barrier coatings. The overall two synthetic process, known as sol-gel chemistry, is illustrated in Scheme 1. First, an alkoxysilane is hydrolyzed by excess water resulting in the substitution of short alkoxide (methyl - $\mathrm{OCH}_{3}$ or ethyl $\mathrm{OCH}_{2} \mathrm{CH}_{3}$ ) groups with hydroxyl groups. The newly formed, reactive hydroxyl groups can then further react through condensation to form Si-O-Si linkages between alkoxysilane molecules. The two byproducts result from this overall reaction, water and alcohol (ROH). Ethanol and methanol are common variations of the alcohol produced. There are a variety of sol-gel systems created through this process that have been

explored extensively in literature. ${ }^{34,35}$ Sol-gel networks are unique among polymers due 
to the inorganic-organic nature of the resultant resin. The inorganic/organic content of these sol-gel resin systems can be controlled through varying amounts of the initial materials used to prepare them. An acid catalyst is commonly needed in these reactions.

a)<smiles>[R20][Si]([R2])([R2])[OH2+]</smiles>

b)<smiles>O[SiH](O)O</smiles><smiles>O[SiH](O)O</smiles>

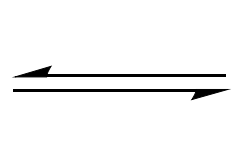

$\mathrm{H}_{2} \mathrm{O}$

Scheme 1. The two-step reaction mechanism found in sol-gel chemistry involving a) hydrolysis of the alkoxysilane and b) condensation of multiple hydrolyzed silane molecules

An alternative reaction similar to sol-gel reactions is the combination of alkoxysilanes with PVOH to form a unique structural network with a variety of different linkages. The reaction mechanism that would occur between these molecules is shown in Scheme 2. It is expected that the integration of silane chemistry into a PVOH resin could help improve water resistance by limiting plasticization by water.<smiles>CC(C)(C)CC(O)C(C)(C)C</smiles><smiles>CC(C)CC(C)O[SiH](O)O</smiles>

Scheme 2. The condensation reaction that would occur between a mixture of PVOH and an alkoxysilane molecule, from which additional reactions could take place to form a network structure 


\subsection{Fillers}

In a conceptual sense, the integration of filler materials is relatively straightforward as the addition of these platy structures can provide diffusivity barrier effects, lowering OTR. Fillers are commonly used in coating formulations to alter gloss and give coatings a translucent appearance due to their low refractive index. Their use in oxygen barrier coatings, however, can be extremely difficult due to the thin nature of these coatings, and limited ability to utilize advanced alignment methods within an industrial production line. The addition of filler materials like talc, kaolinite, montmorillonite, and hectorite clays in small volume fractions of $0-20 \%$ have been used to show improvement in barrier performance. ${ }^{36}$ Even when randomly oriented, these filler particles can affect the barrier ability of a coating. Enhanced barrier performance is obtained when the orientation of the filler is parallel to the coating surface ${ }^{37}$, and no defects are introduced in the resin.

The three most common scenarios that occur when a filler is integrated into a polymer resin are illustrated in Figure 3. The first circumstance produces phase separation, where filler particles remain in small platelets exist separate from the polymeric resin. The improper disperison provides only minor oxygen barrier improvement and risks damaging the initial resin structure via introduction of these clusters. ${ }^{26}$ The second case is that the resin and polymer form an intercalated structure, where multilayers of alternating filler particles and polymeric resin are present throughout the final coating composition. The third outcome is exfoliation, which occurs when filler particles are well dispersed and spaced evenly throughout the polymeric resin. These latter two scenarios can enhance the oxygen barrier properties compared to that of the unaltered resin, but that improvement is dependent on proper dispersion of filler particles, which can be extremely difficult to attain and consistently reproduce. 


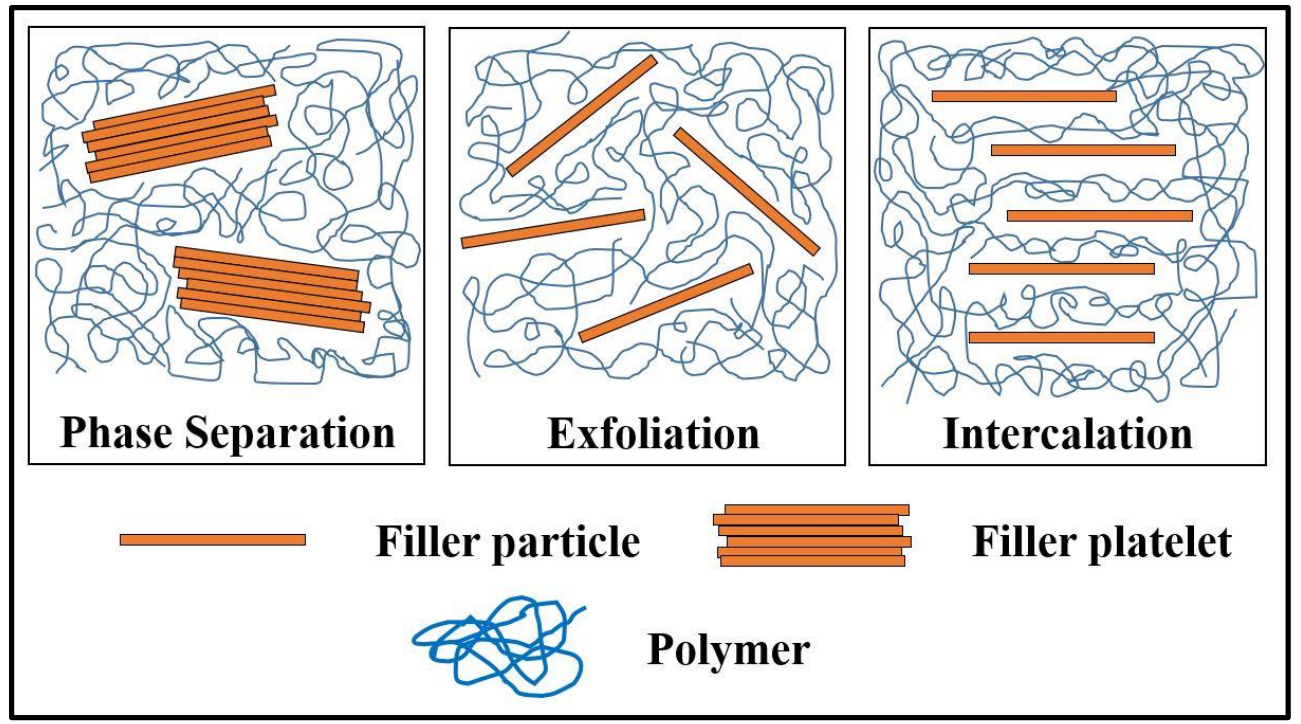

Figure 3. Illustration of the different outcomes of filler integration within an existing polymeric resin

A survey of the literature provides several strong examples of the properties that filler materials can impart on the dry coating if integrated properly. One paper gave decent reduction of oxygen permeability through the addition of montmorillonite into an aqueous PVOH solution, using a rolling technique to help achieve exfoliation of the clay particles. ${ }^{39}$ Other fillers like graphite ${ }^{40}$ and cellulose nanocrystals ${ }^{41}$ can be used to obtain a mix of slight barrier improvements while also changing mechanical properties and water resistance. Even benefits that would be key for food packaging applications such as antimicrobial effects can be imparted through inclusion of silver nanoparticles. ${ }^{42}$ 


\section{METHODS AND MATERIALS}

All coating compositions were prepared in the Cal Poly Kenneth N. Edwards Western Coatings Technology Center Laboratories. Since the composition and specific formulas changed multiple times over the course of the project, the sources of all materials used and the general procedures for the preparation of these different coatings types are summarized here.

\subsection{Materials Used During Coating Preparation}

In preparation of the polyurethane coatings, propylene glycol monomethyl ether acetate, toluene, and methyl isobutyl ketone were supplied by Sigma Aldrich. $p$-xylene was supplied by Fisher Scientific. Acronal 700L was supplied by BASF. Cellulose acetate butyrate (CAB-381-0.1) was supplied by Eastman Chemical Co. Desmophen R221-75 and Desmodur L-75 were supplied by Bayer MaterialScience. Vinylchlorideacetate-alcohol powder was provided by Siegwerk Corporation. All filler materials: Stellar 420, HAR R92, Barrisurf FX, LX, and HX, SoCal U132 and 31 were supplied by Imerys.

As used for preparing the various sol-gel formulations, 1-butanol, pentaerythritol tetraacrylate, and (3-aminopropyl)triethoxysilane were supplied by Sigma Aldrich. Hydrochloric acid (12 N) was supplied by Fisher Scientific. Epon Resin 1001-X-75 was supplied by Hexion. ERL 4221 was supplied by Polysciences Inc. Darocur 1175 was supplied by Ciba. Glycidoxypropyltrimethoxysilane was supplied by Acros Organics. UVI 6992 was provided by Siegwerk Corporation.

For the development of the poly(vinyl alchohol) and vinyltrimethoxysilane coatings, Poval 4-98 was supplied by Kuraray through Siegwerk Corporation. Citric acid, 
vinyltrimethoxysilane, tetraethylorthosilane, and triethylvinylsilane were supplied by the Tokyo Chemical Industry Company. Hydrochloric acid (12 N) was supplied by Fisher Scientific. All materials were used as received without further purification.

\subsection{Preparation of Over-Print Varnish Polyurethane Coatings with Added Fillers}

Plastic THINKY cups were used to mix and transfer the two component over-print varnish (OPV) polyurethane (PU) formulation. The use of the terms components $\mathrm{A}$ and $\mathrm{B}$ describe the groups of materials that are prepared separately. Components A and B were prepared in individual cups prior to mixing them together. Materials were added to THINKY cups in the order listed in the PU formulation using a digital scale to accurately measure out each specified mass. Once all materials were added to each component, the cup was placed into a THINKY AR-100 conditioning mixer for 60 seconds. If fillers were added, component A was sealed with Parafilm and the filler material was dispersed using a Branson 2510 bath sonicator prior to mixing with component B. Dispersing times varied between 20 minutes and 90 minutes depending on the filler used. Once properly mixed, components A and B were then combined, stirred for several minutes with a spatula, and were given an hour induction time before use.

\subsection{Preparation of Sol-Gel Coatings}

THINKY cups were also used in the preparation of the various sol-gel formulations. Depending on whether the system had dual cure capabilities, there were either two or three components. Both components were prepared in individual cups and mixed for 30 minutes using a magnetic stir bar and hot plate. After this mixing period, both components were combined and a drop of concentrated hydrochloric acid was added 
(0.025 mL). Typically a 10-20 minute induction time was allowed before use. If necessary, the third component was added after this curing period and was mixed for 1-2 minutes immediately before use. Thermal curing was conducted by placing the applied film in a Dispatch LDF Series Protocol 3 oven at $60^{\circ} \mathrm{C}$ for 5 minutes. UV curing was accomplished using a LC6 Fusion Conveyor System (“D” lamp I300 Irradiator) operated at a $75 \mathrm{ft} / \mathrm{min}$ belt speed was utilized. This curing rate resulted in an estimated dose of $277 \mathrm{~mJ} / \mathrm{cm}^{2}$ with a $3200 \mathrm{~mW} / \mathrm{cm}^{2}$ peak radiation.

\subsection{Preparation of Poly(vinyl alchohol) \& Vinyltrimethoxysilane Coatings}

A 150 or $250 \mathrm{~mL}$ beaker heated with an oil bath and outfitted with a mechanical stirrer was used for the preparation of the poly(vinyl alchohol) (PVOH)/ vinyltrimethoxsilane (VTMS) blends. First, a stock solution of 18-20 wt.\% aqueous PVOH was prepared. Deionized water was stirred at 100 revolutions per minute and heated to $85-90^{\circ} \mathrm{C}$ before flakes of Poval 4-98 were added in small portions. This solution typically had a yellow hue depending on the amount of Poval added. The PVOH stock was mixed for three hours at the elevated temperature. After equilibrating for at least 24 hours, this PVOH stock solution was then transferred into a reaction beaker and heated to $45-50^{\circ} \mathrm{C}$ before adding any deionized water. VTMS was then added dropwise to the beaker followed by two drops of concentrated hydrochloric acid (approximately 0.05 $\mathrm{mL})$. This solution was mixed for two hours under heat before being cooled and transferred into glass vials. Viscosity was typically tested immediately following the preparation, and the solids percentage was tested after 24 hours along with a second viscosity measurement. 


\subsection{Preparation of Coated Films Using the QD Proofer}

Sample films were prepared using a Harper QD Proofer flexographic doctor-blade printing instrument available in the Cal Poly Graphic Communications Inks \& Substrates Laboratory. The speed on the QD Proofer was kept at a setting of 5 on the speed dial throughout its use. The substrate used was a corona-treated, 12 micron thick poly(ethylene terephthalate) (PET) sheet off of a printing spool provided by Siegwerk Corporation. An anilox roll with a 600 cells per inch (CPI) line screen and a 3.2 billion cubic micron $(\mathrm{BCM})$ cell volume was used in combination with the doctor-blade to deposit coating evenly on the QD Proofer's base roll. Leftover materials were cleaned from all pieces exposed to the coating between every QD Proofer run to prevent residue buildup and skidding.

A $20 \mathrm{~mL}$ vial of the desired coating composition was prepared 24 hours prior to QD Proofer use. Less than a milliliter of coating was required to coat each strip of film. A plastic pipette was used to transfer the mixed coating onto the doctor blade. Once set to 'Proof' the QD Proofer would deposit the coating from the doctor blade, onto the anilox roll, then onto the base roll, and finally onto the PET substrate. The coated region appeared evenly distributed with $7 \mathrm{~cm}$ width, with leftover coating built up at the edges. The PET substrate width, comparatively, was $10 \mathrm{~cm}$, leaving about a third of the film width uncoated on either edge of the substrate. A $32 \mathrm{~cm}$ long strip of the coated PET film was cut, placed on a piece of cardstock, and transferred into an JEIO Tech Lab Companion $\mathrm{ON}-01 \mathrm{E}$ convection oven set at $60^{\circ} \mathrm{C}$ for one hour in order to force cure the sample. In order to obtain similar dried coating thicknesses for the lower viscosity PVOH/VTMS blends, a second coating layer was deposited for these samples. Referred 
to as a 'double-layer' film, the original cured single layer film was fastened on the QD Proofer, another layer of coating was deposited, and the film was subjected to a second curing period.

\subsection{Oxygen Transmission Rate Testing}

All oxygen transmission rate tests were conducted in the Cal Poly Industrial Packaging and Technology Laboratory. Oxygen transmission rate was measured using a MOCON OX-TRAN 2/22 Model L instrument. The units of these OTRs were expressed in milliliters of oxygen gas per square meter of film per day of testing $\left(\mathrm{cc} \mathrm{m}^{-2} \mathrm{day}^{-1}\right)$. Continuous testing mode was utilized with an hour of conditioning time and advanced manually after approximately 12 hours; normally following the general procedure described in ASTM D3985-05. ${ }^{9}$ These tests used a 98\% nitrogen/2\% hydrogen mixture as the carrier gas and oxygen as the test gas at a constant temperature of $23^{\circ} \mathrm{C}$ and pressure of $1 \mathrm{~atm}$. Relative humidity (RH) either was tested at $0 \%$ or $90 \% \mathrm{RH}$.

For each coating composition, two set of tests were run to ensure reproducibility, with each test involving use of both sampling cells of the OX-TRAN instrument, yielding a total of four trials. Occasionally one to three trials were obtained due to damaged samples or malfunctions of the instrument.

Masked films were created for every trial of OTR testing using an aluminum and adhesive template. This template was folded in half to create two connected faces of the mask and cut to the proper size using a metal stencil. The metal stencil and a hammer were used to cutout a consistent $1 \mathrm{~cm}$ radius hole in the middle of both faces of the mask. Any wrinkles or creases were evened out using the edge of the hammer. An approximate 2" x 2" square was removed from the film sample using an exacto knife and examined for 
noticeable defects. The inner plastic backing was removed from both faces of the mask to expose the adhesive backing and the square of film was placed over one face's cutout. The second face was then folded over to enclose the mask with the coated side of the film oriented downwards. In order to mount the samples, Apiezon T grease was used to seal the mask onto the metal half of either of the OX-TRAN instrument's sampling cells with the coating oriented downwards. The second half of the cell was placed underneath the metal half enclosing the film mask in-between.

\subsection{Coating and Film Characterization}

Viscosity profiles were extensively taken toward the later stages of development of the PVOH/VTMS blends. After preparation, the viscosity was monitored approximately every two hours for the first 24 hours and progressively for up to a month after. A TA Instruments Discovery Hybrid Rheometer (DHR-2) was equipped with a $2^{\circ} 40 \mathrm{~mm}$ cone set to a 55 micron gap to measure the shear viscosity. A 60 second test was conducted at a shear rate of $10 \mathrm{~s}^{-1}$ and a temperature of $25^{\circ} \mathrm{C}$, giving viscosity values as a function of time. The 60 second viscosity was used for all reported measurements.

Solids content was determined closely following ASTM D2369-10. Samples of the coatings were weighed prior to being heated in a Dispatch LFD series Protocol 3 oven at $110^{\circ} \mathrm{C}$ for an hour. Each sample was then weighed after oven treatment to quantify the weight loss due to water and solvent evaporation. Aluminum pans were used to hold the samples and a typical 1-2 gram coating sample was spread out over the bottom of each pan.

Film samples were consistently tested for thickness using a simple determination method. A minimum of two $10 \mathrm{~cm}$ by $3 \mathrm{~cm}$ samples were cut out from sample films and 
weighed individually on a digital scale to determine coating weight, using a square piece of aluminum foil below them to prevent static. The length, width, and mass were entered in an Excel sheet, which, along with the estimated density of the coating, were used to determine the coating thickness.

While this thickness determination method seems like an inaccurate method, the thickness obtained from sample to sample with different compositions generally agreed closely with one another. However, to justify that the thicknesses resulting from this procedure were a proper indication of the actual coating thickness, verification by an advanced method was sought after. First, attempts to measure the coating thickness by imaging the cross-section using a Leica DM2500P Polarized Optical Microscope (POM) and FEI Quanta 200 Scanning Electron Microscope (SEM) were conducted. Use of the SEM was provided by the Materials Engineering department. These methods didn't allow for accurate determination of thickness since the cross-section was typical not cleanly cut and differentiation between the coating layer and the substrate was impossible. One time use of a white light interferometer was provided by the Civil and Environmental Engineering department. This spectroscopy method allows for 3D mapping of a sample's surface height profile using the information provided by light wave fronts. The thicknesses obtained through this method on samples of PU and sol-gel coating compositions closely matched the thicknesses determined using our method. 


\section{RESULTS AND DISCUSSION}

At the beginning of the project, a conference call was conducted with the entire team of students, professors, and Siegwerk representatives to distinctly set several key objectives for the envisioned oxygen barrier coating. Several polymeric films were considered as substrates for this project including polyethylene, poly(ethylene terephthalate), and polypropylene. From these choices, PET was selected for use due to its medium oxygen barrier performance, good mechanical properties, high thermal ceiling, and resistance to shrinking. ${ }^{4}$ The barrier coating was to preferably be solventborne to allow for versatile film formation; alternatively a dual cure formulation, or waterborne formulation was desired. The ideal working viscosity was expected to be 50 seconds on a \#2 Zahn cup, which is roughly equivalent to $100-200 \mathrm{mPa}$ s. A high solids percentage was desired to produce a clear, continuous coating with a thickness no greater than 2 microns. The dried, cured film was expected to be water resistant, have no objectionable odor, and be able to resist heat exposure up to $100^{\circ} \mathrm{C}$. The oxygen transmission rate of this coated film at $0 \%$ relative humidity was expected to meet or exceed the performance of leading barrier films. An OTR of $1-10 \mathrm{cc} \mathrm{m}^{-2} \mathrm{day}^{-1}$ is the standard for food packaging applications. ${ }^{24}$ Testing of prepared films at high humidity was a necessary task to test water resistance. Poly(vinyl alchohol), ethylenevinyl alcohol (EVOH), and polyvinyldiene chloride (PVDC) systems were to be avoided ether due to environmental concerns with the chemicals involved or due to the difficulty involved in creating a novel variation of existing coating compositions.

Experimentation began with two coating systems: an industrial high gloss, transparent over-print varnish polyurethane formulation provided by Siegwerk 
Corporation, and sol-gel formulations found commonly in literature studies. As the name suggests, the OPV PU is typically used as a thin, transparent laminate layer put on paper prints. This polyurethane formulation was not expected to provide significant oxygen barrier performance without alteration, since the resin is amphiphilic in nature and doesn't form significant internal structure. The desired oxygen barrier performance was hypothesized to be obtainable through the addition of filler material to the PU formulation. In this system, it was vital to ensure effective dispersion of the filler particles throughout the coating depth to allow for diffusivity barrier effects. If proper integration of the filler was demonstrated, significant reduction of OTR of the coated film was expected when compared to the OTR of the substrate. In contrast, the sol-gel approach would develop dense organic-inorganic networks formed through hydrolysis and condensation reactions. These coatings were expected to have significantly higher oxygen barrier performance than the OPV PU coatings. This prediction was based on the dense resin structure formed in a sol-gel coating that can act as a diffusion barrier for both oxygen molecules and water molecules attempting to permeate through the coating.

\subsection{Polyurethane}

The main components of the PU resin are Desmophen R-221-75, a polyester with a low percentage of hydroxyl groups, and Desmodur L-75, an aromatic polyisocyanate. The reactive functional groups of these two components react to form polyurethane following the general mechanism shown in Scheme 3. The resultant polyisocyante resin has both hydrophobic and hydrophilic regions that are susceptible to oxygen permeation and water plasticization, respectively. This amphiphilic nature in turn limits the oxygen barrier ability that can be obtained due to poor solubility barrier effects. The degree of 
crystallinity formed by the polyurethane resin is largely dependent on the ratio between 'hard' segments of the polyurethane, composed of mainly by aromatic regions with low free volume, and 'soft' segments, composed of flexible hydrocarbon and ester bonds that have high free volume. The low degree of crystallinity expected in this polyurethane formulation thus limits contribution to oxygen barrier from diffusivity barrier effects.

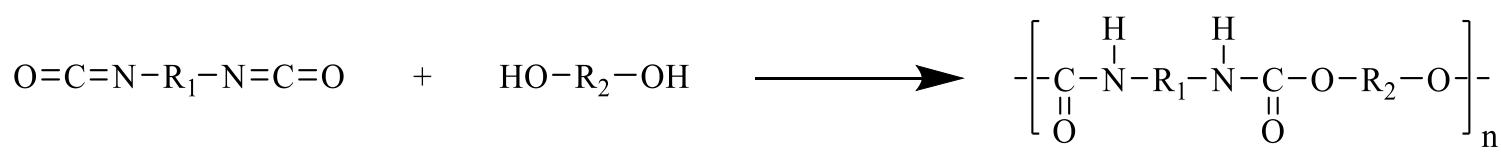

Scheme 3. Typical reaction mechanism between a diisocyanate molecule and a polyol forming a polyurethane

This PU formulation has a moderate non-volatile weight (NVW) of 35\%, otherwise made up of solvents including propylene glycol monomethyl ether acetate (PM acetate), $p$-xylene, methyl isobutyl ketone, and toluene. These providing optimal solubility for the resin components and help control the film formation process. Small amounts of vinylchloride-acetate-alcohol (VAGH) powder, CAB 381-0.5, and Acrynol 700L are added to help control characteristics of the coating such as viscosity, chemical resistance, adhesion, toughness, and hardness. Once solubilized, these molecules have active functional groups that can also be integrated into the network structure of the resin. In low levels ( $1-3$ wt. \% of the dry film composition), filler materials were expected to be fairly easily dispersed through mechanical or hand mixing. ${ }^{36}$ The OPV PU formulation is shown in Table 2, both with and without filler additions. Integration of these filler materials was potentially expected to cause defects in the coating due to poor interactions between the resin and filler particles, and a loss of transparency due to the difference of refractive index between the resin and filler particles. 
Table 2. 35\% NVW OPV polyurethane formulation with and without added filler

\begin{tabular}{|l|r|r|}
\hline \multicolumn{1}{|c|}{$\begin{array}{c}\text { Full Gloss Clear 35\% NVW OPV } \\
\text { Polyurethane Coating }\end{array}$} & Base PU & PU w/ Filler \\
\hline & Weight (g) & Weight (g) \\
\hline COMPONENT I & 0.78 & 0.78 \\
\hline VAGH powder (s) & 1.15 & 1.15 \\
\hline PM acetate (1) & 0.95 & 0.95 \\
\hline $\begin{array}{l}\text { Methyl isobutyl ketone (4-methyl-2- } \\
\text { pentanone) (l) }\end{array}$ & 1.02 & 1.02 \\
\hline p-Xylene (l) & 0.12 & 0.12 \\
\hline CAB 381-0.1 (s) & 9.20 & 9.20 \\
\hline PM acetate (1) & 2.59 & 2.59 \\
\hline $\begin{array}{l}\text { Methyl isobutyl ketone (4-methyl-2- } \\
\text { pentanone) (1) }\end{array}$ & & 2.59 \\
\hline$p$-Xylene (l) & 2.59 & 0.08 \\
\hline $\begin{array}{l}\text { Acronal 700L solution in ethyl acetate } \\
\text { (50 wt.\% s) }\end{array}$ & 0.08 & 11.53 \\
\hline Desmophen R-221-75 (75 wt.\% s) & & 0.14 \\
\hline Filler Material (1 wt.\% of dry coating) & 11.53 & \\
\hline COMPONENT II & 0.00 & 5.93 \\
\hline $\begin{array}{l}\text { Desmodur L-75 in ethyl acetate (75 } \\
\text { wt.\% s) }\end{array}$ & & $\mathbf{4 0 . 1 5}$ \\
\hline Toluene (1) & 5.93 & 35.31 \\
\hline Total (I \& II) & 4.07 & 4.07 \\
\hline Non-volatile Weight (\%) & 35.08 & \\
\hline
\end{tabular}

The main focus in the early stages was to successfully integrate each filler variation into the OPV PU coating, while maintaining a uniform and transparent coating for each system. Several fillers were selected for testing from materials already available in the Kenneth N. Edwards Western Coatings and Technology Center, or that were obtained by Siegwerk Corporation. The fillers that were used are summarized in Table 3 with compositions, particle sizes, and aspect ratios as detailed by the supplier. The Stellar 420 and HAR R92 fillers were talcs that have significantly different median particle diameters. Kaolinite clays, known by tradenames Barrisurf FX, LX, and HX, were 
claimed to disperse well in aqueous media and their varying aspect ratios allow for comparison of how different particles shapes affect the ease of dispersion. Both the talc and kaolinite fillers fall under the classification of a clay, and are provided in the form of platelets of clay particles that require adequate dispersion for most uses. Two calcium carbonate materials, SoCal U132 and 31, were also used as filler materials. These calcium carbonates were the only true nanofillers according to the average median particle. This is an important distinction from the micro-sized clay particles, as the nanofiller may ease the dispersion process. As a result, these calcium carbonate nanofillers could provide larger improvements in OTR.

Table 3. Description of filler material compositions, median particle diameters, and aspect ratios as provided

\begin{tabular}{|c|c|c|c|}
\hline Filler & Chemical Composition & $\begin{array}{l}\text { Median Particle } \\
\text { Diameter }(\mu \mathrm{m})\end{array}$ & $\begin{array}{c}\text { Aspect } \\
\text { Ratio }\end{array}$ \\
\hline Stellar 420 & Talc & 10 & N/A \\
\hline HAR R92 & Talc & $11.3-34.2$ & N/A \\
\hline Barrisurf FX & Kaolinite & $<1$ & $30-35$ \\
\hline Barrisurf LX & Kaolinite & 3 & 60 \\
\hline Barrisurf HX & Kaolinite & 13 & 100 \\
\hline SoCal U132 & $\begin{array}{l}\text { Uncoated Calcium } \\
\text { Carbonate }\end{array}$ & 0.090 & N/A \\
\hline SoCal 31 & $\begin{array}{l}\text { Hydrophobic Coating } \\
\text { around Calcium Carbonate }\end{array}$ & 0.065 & N/A \\
\hline
\end{tabular}

Initially, the filled OPV PU formulations were crudely evaluated by making 3 and 5 mil drawdowns on glass plates. Once coated, the glass plates would be transferred to the oven for an hour at $60^{\circ} \mathrm{C}$ before evaluation. The main two characteristics of the cured PU film that were observed and qualified were the presence of noticeable grainy particles 
within the coating, and the retention of full transparency. After obtaining several cured films with grainy textures and translucent appearance, it became apparent that hand mixing originally used in preparation of the PU formulation was insufficient to properly disperse the filler materials.

Since the THINKY AR-100 conditioning mixer had already been used in the preparation of the OPV PU formulations, hand mixing was replaced by a 1 minute run in the THINKY mixer in an attempt to better disperse the filler within the coating. This approach caused the filler particles to be pulled out of the mixture, accumulating at the bottom of the THINKY cup. This effect was explained by the centrifugal force that the THINKY mixer utilizes in its mixing mechanism, which can force solid materials to settle out. Instead, a Branson 2510 bath sonicator was used to aid in dispersion of the fillers. This instrument emits ultrasound waves through a water filled metal reservoir, which can break apart clustered particles. In order to keep the plastic THINKY cups submerged in the ultrasound bath, metal clamps were utilized, attached to an adjacent ring stand. The THINKY cups were sealed with Parafilm to prevent agitated water from splashing into the formulation.

For each filled PU system, aliquots of the sonicated coatings were taken at regular intervals for up to 90 minutes to determine how much ultrasound sonication was necessary to obtain good dispersion and coating appearance. After sonication times of 20 - 90 minutes, visible improvement of filler dispersion was achieved across all of the filled PU systems. The quality of these coatings were ranked on a 1 to 5 scale. A value of 1 signified a poorly dispersed coating with translucent appearance and noticeably grainy texture. At the other end of the scale, a value of 5 meant high dispersion of filler particles 
was obtained yielding a homogenous and transparent coating. All of the PU coatings were translucent when applied to the glass plates, though, once dried and cured they reverted to near transparency. The summary of these results are illustrated in Table 4, organized by filler material. The optimal sonication times for each filler material are indicated by a bolded number. For example, the Stellar 420 filler gave significantly improved dispersion after 20 minutes of sonication, while 90 minutes was required to achieve the same level coating quality for the larger HAR H92 talc particles. Sonication times resulting in a quality value of $4-5$ for a given filler were considered to provide good enough particle dispersion and coating quality to justify preparing actual films on the QD Proofer using that filled PU system.

Table 4. Summary of sonication times necessary to achieve proper filler dispersion and coating quality for each filler material

\begin{tabular}{|l|c|c|c|c|c|}
\hline \multirow{2}{*}{$\begin{array}{c}\text { Material } \\
\text { Used }\end{array}$} & \multicolumn{5}{|c|}{ Sonication Time (minutes) } \\
\cline { 2 - 6 } & 0 & 20 & 40 & 60 & 90 \\
\hline Stellar 420 & 2 & $\mathbf{4 . 5}$ & & 5 & \\
\hline HAR H92 & 2 & 2.5 & 3 & 3.5 & $\mathbf{4 . 5}$ \\
\hline Barrisurf FX & 1 & 2 & $\mathbf{4}$ & & \\
\hline Barrisurf LX & 1 & 2 & 2.5 & & \\
\hline Barrisurf HX & 1 & 3 & $\mathbf{5}$ & & \\
\hline SoCal 31 & 1 & $\mathbf{4}$ & & 4.5 & \\
\hline SoCal U132 & 1 & 3 & & $\mathbf{4}$ & \\
\hline
\end{tabular}

From the talc compositions, it was immediately apparent that the Stellar 420 talc produced well-dispersed films even at low sonication times of 20 minutes. In comparison, the HAR H92 particles didn't allow for proper dispersion, unless sonication times longer than 60 minutes were used. The HAR H92 talc particles have the highest median 
diameter of any of the fillers at 34.2 microns, the high end of the range specified by the provider. This diameter is over three times as long as the second longest filler particle, and may be the main reason poor dispersion results from integration of the HAR R92 particles compared to Stellar 420 particles. Since the coating layers produced using the QD Proofer were 1-2 microns thick, it is easy to speculate that larger filler particles are harder to properly align within the coating layer, especially without causing defects to occur. Due to significantly ease of dispersion and better coating quality, the Stellar 420 filler was designated for further testing out of the two talc compositions.

The Barrisurf kaolinite clays provided by Siegwerk had a range of aspect ratios that could be used to compare the effect that different particle shapes have on the ability to disperse individual particles. Barrisurf FX, LX, and HX have an increasing median diameter and aspect ratio in the order listed. Interestingly, the three kaolinite grades didn't provide a direct relationship between particle size and quality of dispersion. The smallest median particle, Barrisurf FX, and the largest median particle, Barrisurf HX, showed similar dispersion and coating transparency at an optimal sonication time of 40 minutes. The Barrisurf HX kaolinite particles have a median particle diameter of 13 microns, similar to the size of Stellar 420 talc particles. For this reason, Barrisurf HX was picked to represent the kaolinite filler composition, so that its OTR could be directly compared with the OTR obtained from films of the Stellar 420 PU system.

Both of the SoCal calcium carbonate fillers integrated fairly readily into the PU formulation, showing optimal dispersion after 60 minutes of sonication. The SoCal 31 filler was selected over its counterpart due to a lower sonication time of 20 minutes required to achieve particle dispersion. 
Use of the QD Proofer to prepare coated films gave higher reproducibility from sample to sample than films prepared using the drawdown bar on glass plates.

Occasionally the base roll would skid while depositing the coating layer, or residue from a previous run would cause consistent defects in the coated region. These films were discarded since damage to the coating could cause major increases in OTR. Both of these problems could be remedied, either through adding grease to portions of the base roll, or by cleaning the pieces of the doctor blade, anilox roll, and base roll more extensively between uses. Films prepared on the QD Proofer had uniform thickness across the coated area, typically $1-3$ microns thick, varying slightly between compositions. The thinner films didn't exhibit any problems with increased translucency, since the path length taken by light through the coating isn't long enough for a hiding effect to occur. None of these films showed the grainy texture observed in the thicker glass drawdown coatings.

Further analysis of the QD Proofer films used polarized optical microscopy to image sections at $5 \mathrm{x}$ magnification. At this magnification, the polyurethane coating appeared to have a moderate frequency of air pockets and defects. However, no aggregation or clumping of filler particles was noticed. The wrinkled texture seen in the base PU film was not observed in the $1 \mathrm{wt} . \%$ Stellar 420 PU film. Representative images taken on the POM of unfilled and filled PU formulations are shown in Figure 4 for comparison. 

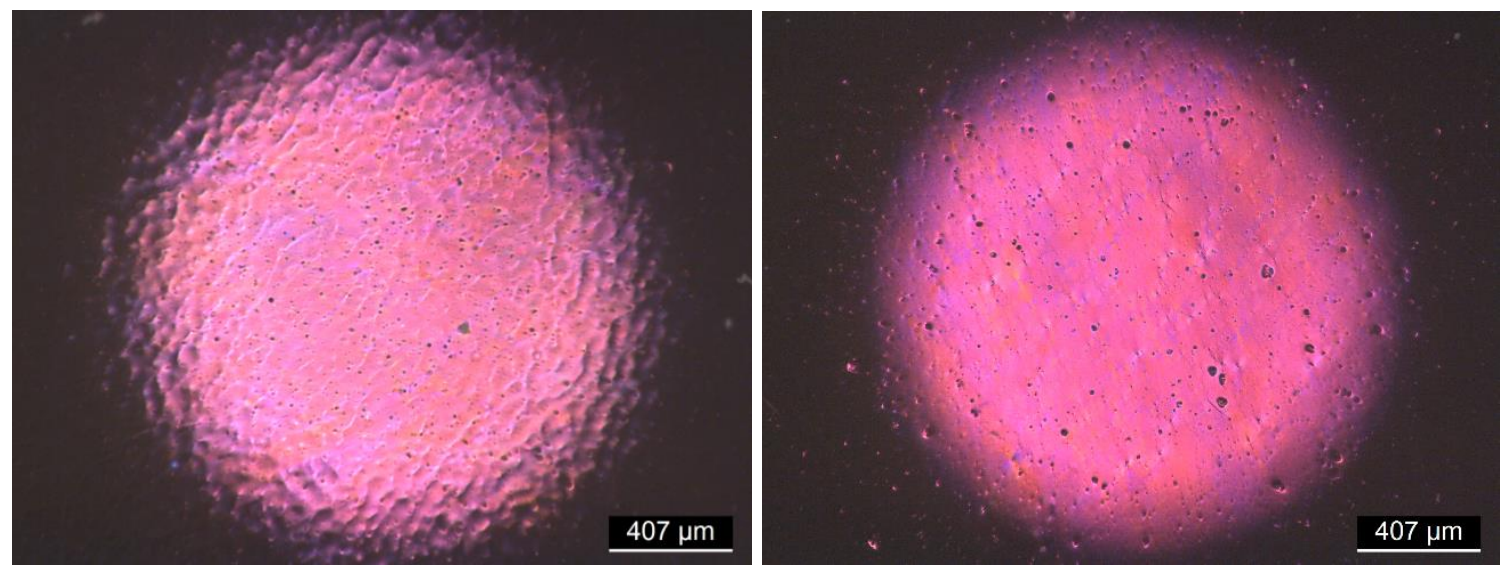

Figure 4. POM image of unfilled (left) and 1 wt.\% Stellar 420 (right) OPV 50\% NVW PU films at $5 x$ magnification

Masked samples were prepared to begin oxygen transmission rate testing on the MOCON OX-TRAN instrument as soon as films of the unaltered and filled OPV PU formulation had been prepared on the QD proofer. Before testing the filled PU films, reference OTRs at $0 \%$ relative humidity were measured for the 12 micron thick, uncoated PET substrate, as well as a coated film of the base PU formulation. For the bare substrate and OPV PU coated film, OTR values of $156.13 \mathrm{cc} \mathrm{m}^{-2}$ day $^{-1}$ and $154.96 \mathrm{cc} \mathrm{m}^{-2}$ day $^{-1}$ ( $0.75 \%$ reduction), respectively, were obtained from an average of four samples per film type. These two oxygen transmission rates established a baseline of approximately $155 \mathrm{cc}$ $\mathrm{m}^{-2}$ day $^{-1}$, and confirmed that the unfilled PU coating doesn't display significant oxygen barrier performance.

The desired improvement wasn't shown for any of the filler compositions, despite the expectation that these filler materials would integrate well into the PU formulation helping to lower the OTR of the PET substrate. The largest decrease in OTR was seen with the addition of 1 wt. $\%$ of Stellar 420 , where an OTR of $132.98 \mathrm{cc} \mathrm{m}^{-2}$ day $^{-1}(12.5 \%$ reduction) for the coated film. Since nearly $100 \%$ reduction is required to obtain OTRs 
similar to prominent oxygen barrier coatings, these formulations were far from the barrier performance expected. The OTRs of the other filler compositions are listed in Table 5, along with the coating thickness. Due to time constraints, the OTRs of HAR R92 and SoCal 31 filled PU films were not tested.

Table 5. Oxygen transmission rates and related thicknesses for the $30 \%$ NVW PU formulations

\begin{tabular}{|l|c|c|}
\hline \multicolumn{1}{|c|}{ Coating Composition } & $\begin{array}{c}\text { Coating } \\
\text { Thickness }(\boldsymbol{\mu m})\end{array}$ & OTR at 0\% RH $\left(\mathbf{c c ~ m}^{-2}\right.$ day $\left.^{-1}\right)$ \\
\hline PET Substrate & N/A & $156.13 \pm 10.75$ \\
\hline PET-PU base & 1.39 & $154.96 \pm 4.80$ \\
\hline PET- PU Stellar 420 (1 wt.\%) & 1.75 & $132.98 \pm 1.71$ \\
\hline PET- PU Stellar 420 (3 wt.\%) & 1.25 & $146.32 \pm 1.63$ \\
\hline PET- PU Stellar 420 (5 wt.\%) & 1.21 & $136.68 \pm 7.58$ \\
\hline PET- PU HAR R92 (1 wt.\%) & 1.31 & $142.51 \pm 0.55$ \\
\hline PET- PU Barrisurf FX (1 wt.\%) & 1.24 & $145.52 \pm 8.54$ \\
\hline PET- PU Barrisurf HX (1 wt.\%) & 1.52 & \\
\hline PET- PU SoCal 31 (2 wt.\%) & 1.16 & \\
\hline
\end{tabular}

The lack of improvement seen by these filler additions could be attributed to a variety of reasons. The most likely contribution comes from the inability to properly intercalate or exfoliate the filler into the resin. Without this thorough dispersion, filler particles are not expected to align within the coating, limiting their ability to provide substantial diffusivity barrier effects. The slight OTR reduction observed can still be explained simply due to the integrated filler materials that provide low diffusivity barrier effects, even if they are only randomly or partially oriented with the coating.

The most significant result coming from the preparation of these filled PU films was the average dry coating thickness using the specified anilox roll. Thickness of these films 
was consistently an average value of 1.35 microns, within the target thickness specifications established for the project. Since other coating resin compositions were predicted to have similar solids percentages, viscosities, and film formation processes, thicknesses of any films prepared on the QD Proofer were expected to remain within 1 2 microns. Thicknesses obtained throughout the project confirm this expectation.

In response to the inadequate OTR reduction observed for the $35 \% \mathrm{NVW}$ filled PU formulations, the PU formulation was adjusted to raise the non-volatile weight from $35 \%$ to 50. This change to the formulation was expected to increase the dry coating thickness. By increasing the thickness of the coating layer, it was thought that any defects resulting in the thinner films would be less likely to occur from a thicker deposition. These thicker coatings may also allow more depth for the filler particles to effectively orient, without causing defects near the surface of the coating layer. The adjusted 50\% NVW PU formulation can be seen in Table 6, which differs from the 35\% NVW PU formulation in the amount of each solvent used. 
Table 6. 50\% NVW OPV PU formulation with and without filler

\begin{tabular}{|l|r|r|}
\hline \multicolumn{1}{|c|}{$\begin{array}{c}\text { Full Gloss Clear 50\% NVW OPV Polyurethane } \\
\text { Coating }\end{array}$} & Base PU & PU w/ Filler \\
\hline COMPONENT I & Weight (g) & Weight (g) \\
\hline Vinyl VAGH powder (s) & 0.78 & 0.78 \\
\hline PM acetate (1) & 1.15 & 1.15 \\
\hline Methyl isobutyl ketone (4-methyl-2-pentanone) (1) & 3.54 & 3.54 \\
\hline p-Xylene (1) & 1.02 & 0.12 \\
\hline CAB 381-0.1 (s) & 0.12 & 0.08 \\
\hline Acronal 700L solution in ethyl acetate (50 wt.\% s) & 0.08 & 0.14 \\
\hline Filler (Talc) (1 wt.\% dry coating) & 0.00 & 11.53 \\
\hline Desmophen R-221-75 (75 wt.\% s) & 11.53 & \\
\hline COMPONENT II & & 5.93 \\
\hline Desmodur L-75 in ethyl acetate (75 wt.\% s) & 5.93 & 4.07 \\
\hline Toluene (1) & 4.07 & $\mathbf{2 8 . 3 6}$ \\
\hline Total (I \& II) & 49.80 & 50.05 \\
\hline Non-volatile Weight (\%) & & 0.22 \\
\hline
\end{tabular}

The change in NVW had little effect on the preparation of the coatings or films but did show a noticeable increase in viscosity to $500 \mathrm{mPa}$.s. The viscosity of the $35 \% \mathrm{NVW}$ PU coatings was never directly measured, but was estimated to be in the 100-300 mPa.s range. The thickness of these coatings was, on average, slightly higher than the $30 \%$ NVW PU film samples, but the quality of the coatings didn't appear different. Again, the OTR testing for these 50\% NVW PU films came back with poor oxygen barrier performance (Table 7). The OTR for the 50\% NVW PU film was $154.09 \mathrm{cc} \mathrm{m}^{-2}$ day $^{-1}$ (1.31\% reduction), closely matching the performance of the $30 \%$ NVW PU film with an OTR of $154.96 \mathrm{cc} \mathrm{m}^{-2}$ day $^{-1}$. The addition of $2 \mathrm{wt} . \%$ Stellar 420 to the $50 \%$ NVW PU 
formulation had no significant effect on the OTR compared to the that of the base PU film. These results strongly suggest that the limited reduction of OTR for these filled OPV PU coatings was due to improper dispersion and alignment of the filler particles within the coating layer.

Table 7. Oxygen transmission rates and thicknesses obtained for the 50\% NVW PU formulations

\begin{tabular}{|c|c|c|}
\hline Coating Composition & Thickness ( $\mu \mathrm{m})$ & $\begin{array}{c}\text { OTR at } 0 \% \text { RH } \\
\left(\text { cc } \text { m }^{-2} \text { day }^{-1}\right)\end{array}$ \\
\hline PET-PU Base & 1.77 & $154.09 \pm 4.38$ \\
\hline PET-PU Stellar 420 (2 wt.\%) & 1.77 & 155.00 \\
\hline
\end{tabular}

From these results, it was clear that having a resin structure with poor oxygen barrier ability wasn't going to achieve high reduction in OTR without introducing complex dispersion methods. The biggest drawback was an inability to qualify the extent of dispersion of the filler particles and their orientation in the cured coating. No solutions to this problem were thought of that could fit the specifications of the project and enable use this system moving forward given the up-scaling capabilities required of it.

\subsection{Sol-Gel}

The sol-gel formulations took a different approach toward improving the oxygen barrier performance than the filled OPV polyurethane coatings. Rather than introducing a platy filler into the existing low barrier resin to achieve a strong oxygen barrier coating, sol-gel chemistry was used to create a strong network resin structure capable of providing diffusivity barrier effects. If sol-gel resins demonstrated low OTRs, it was thought that filler materials could potentially be integrated into this high barrier resin, as well, to 
further reduce OTR. This would require additional investigation into dispersion of the filler of course. Four sol-gel systems were designed and prepared while investigation into the filled over-print varnish polyurethanes was also happening.

The key component behind these sol-gel systems are alkoxysilanes. Alkoxysilanes comprise one to four Si-O-R linkages about the central $\mathrm{Si}$ atom are found in one molecule. The R group(s) are alkyl groups, such as methyl $\left(-\mathrm{CH}_{3}\right)$ or ethyl $\left(-\mathrm{CH}_{2} \mathrm{CH}_{3}\right)$. Some examples of alkoxysilane molecules include tetraethylorthosilane (TEOS), (3glycidoxypropyl)trimethoxysilane (GPTMOS), (3-aminopropyl)triethoxysilane (APTS), vinyltrimethoxysilane, and triethoxyvinylsilane (TEVS), which are shown along with other common alkoxysilanes in Figure 5.

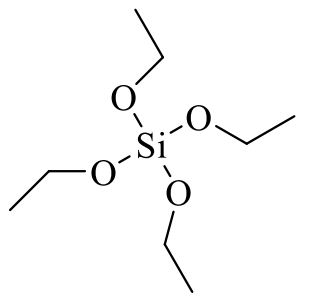

TEOS

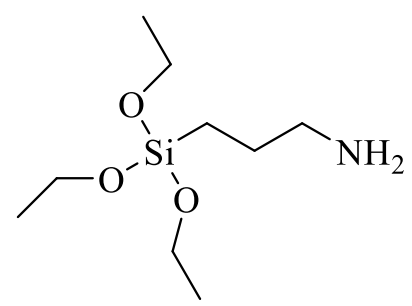

APTS

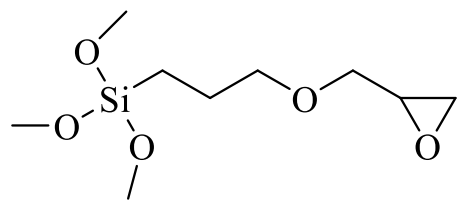

GPTMOS

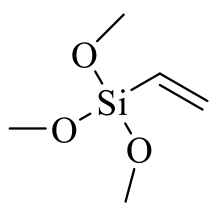

VTMS

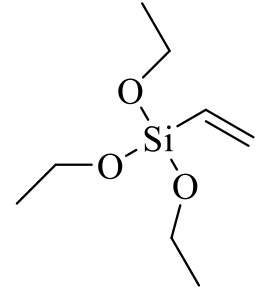

TEVS

Figure 5. Chemical structures of several commonly used alkoxysilane molecules 
The reaction between alkoxysilane molecules occurs in a two-step reaction mechanism. In the first step, the alkoxysilanes undergo a hydrolysis reaction involving water and an acid catalyst. This hydrolysis reaction cleaves silyl ether groups (Si-O-R), which are immediately replaced with hydroxide groups. This causes each alkoxysilane arm of the molecule to be converted into a reactive silanol (Si-O-H) which can react with another hydrolyzed alkoxysilane to form a Si-O-Si linkage. A different crosslinking molecule can be added into these sol-gel systems that has reactive groups such as amines, epoxides, or alcohols, that can covalently bond with silanol groups once the alkoxysilane undergoes hydrolysis. These reactions continue extensively until the majority of molecules have been integrated into a network resin structure.

Significant testing was done with each composition in order to achieve and optimize the curing time and quality of the resulting coatings. Varying the mole ratios of the initial reagents helped control reaction rates and the inorganic-organic content of the resin.

Often, with increasing inorganic content, the mixture would rapidly form an opaque gel. The finalized formulations for these sol-gel systems had variable working times before gelation occurred. Batches were prepared immediately preceding film preparation.

The earliest sol-gel formulation prepared was a system composed of (3aminopropyl)triethoxysilane and Epon Resin 1001-X-75. Epon Resin 1001-X-75 is an aromatic resin with epoxide functionality allowing it to crosslink with another molecule such as APTS ${ }^{43}$ Thermal curing methods were used with this initial sol-gel formulation. The formulation used for this APTS/Epon sol-gel coating is shown in Table 8. Optimization of the APTS/Epon molar ratios was important in controlling the curing time associated with the formulation. 
Table 8. Sol-Gel formulation utilizing APTS and Epon 1001-X-75

\begin{tabular}{|c|c|c|}
\hline \multirow[t]{2}{*}{ Material Used } & Base Sol-Gel & $\begin{array}{l}\text { Sol-Gel with Added } \\
\text { Water }\end{array}$ \\
\hline & Weight (g) & Weight (g) \\
\hline APTS & 1.65 & 1.65 \\
\hline $\begin{array}{l}\text { Epon Resin 1001-X-75 in } \\
\text { xylene }\end{array}$ & 4.90 & 4.90 \\
\hline 1-butanol & 1.30 & 1.30 \\
\hline Water & 0.00 & 0.50 \\
\hline TOTAL & 7.85 & $\overline{8.35}$ \\
\hline \multicolumn{3}{|l|}{ Dry Films Properties } \\
\hline Non-volatiles by Weight & $59.70 \%$ & $56.11 \%$ \\
\hline $\begin{array}{l}\text { Inorganic Composition in } \\
\text { Film by Weight }\end{array}$ & $21.55 \%$ & $21.55 \%$ \\
\hline
\end{tabular}

This APTS/Epon formulation was found to have a working lifetime of approximately 4 hours. After this period, the viscosity raised past a critical level, forming an opaque gel. The addition of water into the formulation was shown to speed up the curing rate. How the lifetime would differ with varying quantities of water was hard to accurately predict. Once these sol-gel coatings were formulated, they were given a 30 minute induction time prior to film preparation on the QD Proofer. Thermal curing at $65^{\circ} \mathrm{C}$ for an hour was enough to progress these sol-gel films to a dry to the touch state. The average thickness associated with this formulation was 2.06 microns.

The second sol-gel formulation followed the same basic principle established in the first formulation, but used a cycloaliphatic epoxide resin instead of an aromatic epoxide resin. The cycloaliphatic epoxide resin used was ERL 4221. Long hydrocarbon chain segments from the epoxide resin can cause a loss in mechanical properties due to the flexible nature arising from this structure. ${ }^{44}$ The inclusion of these long alkyl chains into 
the resin structure may also hinder oxygen barrier due to an increased oxygen solubility in the resin. The APTS/ERL 4221 sol-gel formulation is given in Table 9.

Table 9. Sol-Gel formulation utilizing APTS and ERL 4221

\begin{tabular}{|l|r|r|}
\hline \multicolumn{1}{|c|}{ Material Used } & Weight (g) & Non-volatile Weight (g) \\
\hline APTS & 1.65 & 1.01 \\
\hline Water & 0.50 & 0.00 \\
\hline Butanol & 1.30 & 0.00 \\
\hline ERL 4221 & 1.22 & 1.22 \\
\hline Total & $\mathbf{4 . 6 7}$ & $\mathbf{2 . 2 3}$ \\
\hline Dry Films Properties & \multicolumn{3}{|c|}{$47.75 \%$} \\
\hline Non-volatiles by Weight & $45.29 \%$ \\
\hline $\begin{array}{l}\text { Inorganic Composition in } \\
\text { Film by Weight }\end{array}$ & \multicolumn{3}{|c|}{} \\
\hline
\end{tabular}

Film preparation on the QD Proofer was never done using this formulation, but drawdowns were prepared using a 5 mil drawdown bar on a glass plate. This APTS/ERL 4221 sol-gel formulation took longer to reach a dry to touch state, and the glass drawdowns needed heating in the oven at $65^{\circ} \mathrm{C}$ for 24 hours to ensure complete curing. The films prepared following this formulation were clear and continuous with a similar working lifetime to the APTS/Epon sol-gel formulation of approximately 4 hours. In industry, shorter thermal treatments can help significantly reduce cost during preparation. For this reason, the curing times necessary for this coating are undesirable.

Dual cure sol-gel formulations were also of interest, since the utilization of UV curing or thermal curing is possible. The ability to cure the films within seconds via UV radiation can be easily integrated into a film printing line, eliminating problems associated with longer thermal curing periods. The first dual cure sol-gel formulation 
(Table 10) attempted was modeled around APTS and TEOS molecules that form the basis of the resin. Pentaerythritol tetraacrylate (PTOL) was also included in this formation for its acrylate group functionality. These acrylate groups can be used to extend the sol-gel network structure via Michael addition reactions with amine functionality as well as through photo-induced free-radical polymerization when paired with a photoinitiator package, like Darocur $1173 .{ }^{45} \mathrm{UV}$ exposure can provide the energy to catalyze free-radical formation, which rapidly react causing a tight chemically-bonded network to form within the coating. The extensive resin structure eventually formed from these three molecules was expected to give high diffusivity barrier effects to reduce the OTR of the substrate.

Compared to the thermal cure sol-gel formulations, the coated film quality of the TEOS/APTS/PTOL mixture was no different than those previously tested on the QD Proofer. A potential drawback to this formulation is that, once mixed, it only has a working lifetime of $10-20$ minutes before gelation occurs. Working lifetime, or pot life, is a term used to describe the time period in which the mixture's composition and condition are acceptable for use. Having a working lifetime of at least 8 hours accommodates use throughout a work shift, which is typically standard in industry. The UV cure method provided films that didn't noticeably wrinkle, which is a common problem with UV curing due to the high rate of crosslinking that results from the process. Thermal curing was also improved compared to the APTS/Epon and APTS/ERL 4221 coatings only taking about 10 minutes in an oven at $65^{\circ} \mathrm{C}$. Thicknesses around 1.39 microns were observed for the TEOS/APTS/PTOL films. 
Table 10. Sol-gel formulation utilizing TEOS, APTS, and PTOL

\begin{tabular}{|l|r|r|}
\hline \multicolumn{1}{|c|}{ Material Used } & Weight (g) & \multicolumn{2}{c|}{$\begin{array}{c}\text { Non-volatile } \\
\text { Weight (g) }\end{array}$} \\
\hline TEOS & 2.5 & 0.72 \\
\hline Water_TEOS & 0.86 & 0.00 \\
\hline Butanol_TEOS & 0.88 & 0.00 \\
\hline HCl, conc & 0.03 & 0.00 \\
\hline APTS & 2.5 & 1.53 \\
\hline Water_APTS & 0.75 & 0.00 \\
\hline Butanol_APTS & 1.03 & 0.00 \\
\hline HCl, conc & 0.03 & 0.00 \\
\hline PTOL & 1.2 & 1.20 \\
\hline Darocur 1173 & 0.1 & $\mathbf{3 . 5 5}$ \\
\hline Total & $\mathbf{9 . 8 8}$ & \\
\hline Dry Films Properties & 35.10 \\
\hline Non-volatiles by Weight & \multicolumn{2}{|c}{} \\
\hline Inorganic Composition in & \multicolumn{2}{|c|}{} \\
Film by Weight & \multicolumn{3}{|c|}{} \\
\hline
\end{tabular}

A second dual cure sol-gel formulation was also developed that followed a photoinduced cationic polymerization UV cure route, instead of a photo-induced free-radical polymerization route. This formulation used TEOS and GPTMOS as components of the base sol-gel structure. The change from APTS to GPTMOS provides additional epoxide functionality to the system, complementing the cycloaliphatic epoxide, ERL 4221. Epoxide rings in the GPTMOS and ERL 4221 molecules can be opened and integrated into the sol-gel resin through cationic ring-opening polymerization. Inclusion of a UVI 6992 photoinitator package can initiate this cationic propagation when the film is exposed to $\mathrm{UV}$ radiation. This formulation can be observed in Table 11 . 
Table 11. Sol-gel formulation utilizing TEOS, GPTMOS, and ERL 4221

\begin{tabular}{|l|r|r|}
\hline \multicolumn{1}{|c|}{ Material Used } & Weight (g) & \multicolumn{2}{c|}{$\begin{array}{c}\text { Non-Volatile } \\
\text { Weight (g) }\end{array}$} \\
\hline TEOS & 6.45 & 1.86 \\
\hline GPTMOS & 3.60 & 2.55 \\
\hline Water & 3.37 & 0.00 \\
\hline HCl, conc & 0.06 & 0.00 \\
\hline ERL 4221 & 2.04 & 0.00 \\
\hline UVI 6992 & 0.32 & $\mathbf{6 . 4 5}$ \\
\hline TOTAL & $\mathbf{1 5 . 8 4}$ & \\
\hline Dry Films Properties & & \\
\hline Non-volatiles by Weight & $40.72 \%$ & \\
\hline $\begin{array}{l}\text { Inorganic Composition in } \\
\text { Film by Weight }\end{array}$ & $41.19 \%$ & \\
\hline
\end{tabular}

Like the other sol-gel coatings, no defects or opacity problems were observed in the coated films prepared on the QD Proofer. Both thermal and UV cure methods provided rapid curing of films similar to the TEOS/APTS/PTOL sol-gel films. The average thickness of the TEOS/GPTMOS/ERL 4221 films prepared on the QD Proofer was 1.24 microns.

While the sol-gel films gave slightly better results than the filled OPV PU films, the resultant OTRs were still nowhere near the oxygen barrier performance desired for the project. Of the sol-gel compositions tested, the APTS/Epon films showed the best improvement in OTR compared to the bare PET value of $156.13 \pm 10.75 \mathrm{cc} \mathrm{m}^{-2}$ day $^{-1}$. The OTR obtained for the APTS/Epon sol-gel formulation was $143.55 \mathrm{cc} \mathrm{m}^{-2} \mathrm{day}^{-1}(8.1 \%$ 
reduction) at $0 \% \mathrm{RH}$. The reduction of OTR provided by the $1 \mathrm{wt} \%$ Stellar $420 \mathrm{PU}$ coating of $12 \%$ was still better than the reduction from the APTS/Epon coating. All the sol-gel film OTRs are illustrated in Table 12.

Table 12. Oxygen transmission rate and properties for the sol-gel systems

\begin{tabular}{|l|c|c|}
\hline \multicolumn{1}{|c|}{ Coating Composition } & Thickness $(\boldsymbol{\mu m})$ & $\begin{array}{c}\text { OTR at 0\% RH } \\
\left(\mathbf{c c ~}^{\mathbf{- 2}} \mathbf{d a y}^{\mathbf{1}}\right)\end{array}$ \\
\hline PET - APTS/Epon Resin Sol-Gel & 2.03 & $143.55 \pm 5.19$ \\
\hline PET - APTS/ERL 4221 Sol-Gel & & \\
\hline PET - TEOS/APTS/PTOL Sol-Gel & 1.39 & 144.1 \\
\hline PET -TEOS/GPTMOS/ERL 4221 Sol-Gel & 1.24 & $163.37 \pm 14.82$ \\
\hline
\end{tabular}

The sol-gel chemistry was expected to provide a mix of organic-inorganic content that would result in a stronger oxygen barrier resin compared to the polyurethane formulations. It is difficult to predict whether the improvement in the coating resin structure would have a stronger impact on oxygen barrier than the integration of filler materials. If free of holes and defects, however, development of the internal resin could provide both solubility and diffusivity barrier effects. Polarized optical microscopy was utilized on the sol-gel films to see if holes and defects were present. A POM image of the TEOS/GPTMOS/ERL 4221 film is shown in Figure 6. Compared to the POM of the filled OPV PU films, the sol-gel films had less texturing and a similar amount of holes. From this appearance, there are no real indications of significant film defects in the solgel formulations. Other spectroscopy methods, such as scanning electron microscopy or Fourier transform infrared spectroscopy, could provide further insight to why these solgel formulations provided such poor oxygen barrier performance. 
Figure 6. POM image of the TEOS/GPTMOS/ERL 4221 films at 5x magnification

It is difficult to explain why these sol-gel formulations didn't perform as well as some successful oxygen barrier coatings demonstrated in literature. The procedure behind making these sol-gel coatings isn't extremely complex, however, variation of molar ratios between components of each formulation seems to have a strong impact on how fast and to what extent the sol-gel reaction occurs. Given the OTR results obtained between the polyurethane and sol-gel oxygen barrier coatings, it was apparent that these approaches weren't close to achieving the $1-10 \mathrm{cc} \mathrm{m}^{-2}$ day $^{-1}$ target oxygen transmission rate.

\subsection{Poly(vinyl alchohol) \& Vinyltrimethoxysilane}

After the polyurethane and sol-gel formulations failed to show significant improvement desired, the project team tried to gain a more general picture of the potential systems that provide high oxygen barrier performance. Information related to oxygen barrier coatings, barrier coatings, oxygen permeation, and oxygen transmission rate was gathered from references found during early research stages and through studies published in scientific journals. This information was compiled into detailed tables 
outlining synthetic conditions, curing conditions, oxygen permeability, and oxygen transmission rates for various barrier coating systems. A particular interest was taken in studies that reported oxygen transmission rates since the relative improvement between the coated and uncoated films could be compared for these systems. Considering only examples of oxygen barrier coatings that showed improvements of $\sim 85 \%$ or more, thirteen papers were selected that met that qualification. Several of these articles could be ruled out as future directions for this project, either due to use of expensive technologies that were not available on the Cal Poly campus, or insufficient detail in their procedures to effectively replicate and develop the system

One paper stood out among others as a potential system for this project because of a simple preparation procedure, data suggesting significant reduction in OTR, and a similar nature to the sol-gel systems previously tested. ${ }^{46}$ This oxygen barrier coating was prepared through a two component, solution-based system utilizing an acid catalyst. The concept was to combine sol-gel chemistry into the typical poly(vinyl alcohol) structure, to create an intrinsically strong oxygen barrier resin. The general reaction mechanism closely resembles that of sol-gel systems, since PVOH has pendant hydroxyl groups that can undergo condensation reactions with hydrolyzed VTMS molecules. For the sol-gel chemistry to occur, hydrolysis of silyl ether (Si-O-R) linkages in the VTMS molecule occurs under acidic conditions, forming reactive silanol (Si-O-OH) moieties. Condensation reactions can occur between hydroxyl groups forming $\mathrm{Si}-\mathrm{O}-\mathrm{Si}$ or $\mathrm{Si}-\mathrm{O}-\mathrm{C}$ linkages. The potential of condensation with PVOH pendant hydroxyl groups introduces more variation in the resin structure, while still maintaining a tight connected network. The vinyl group on each VTMS molecule isn't predicted to react with the rest of the 
resin, instead being incorporated into the internal structure potentially aiding with adhesion to certain substrates. ${ }^{46}$

The PVOH resin structure that results oxygen has high polarity and moderate crystallinity, which influence solubility barrier and diffusivity barrier effects, respectively. The addition of vinyltrimethoxysilane in minor weight fractions $(5-20$ wt.\%) to an aqueous poly(vinyl alchohol) mixture was shown to provide water resistance and slight reduction in OTR from that of pure $\mathrm{PVOH} .{ }^{46} \mathrm{~A}$ formulation utilizing this concept was considered the best direction to take to achieve both high oxygen barrier performance and retention of OTR at high humidity.

Experimentation into the preparation of this PVOH/VTMS mixture was started following the rough outline provided by the study. Poly(vinyl alchohol) was provided by Siegwerk in the form of Poval 4-98, which came as slightly yellow, crystalline flakes. This polymer was dissolved into deionized water to prepare $16-22$ wt.\% stock solutions of aqueous PVOH with viscosities between 100 - $800 \mathrm{mPa}$.s. In order to fully dissolve the $\mathrm{PVOH}$, the water was heated to approximately $90^{\circ} \mathrm{C}$, near the glass transition temperature of PVOH, and mixed at $100 \mathrm{rpm}$ for 3 hours using a mechanical mixer. This stock solution was cooled and stored overnight before being used in the subsequent PVOH/VTMS preparation. VTMS, a colorless liquid, was added dropwise to the heated $\left(50^{\circ} \mathrm{C}\right)$ stock PVOH solution followed by two drops of concentrated $12 \mathrm{~N}$ hydrochloric acid $(\mathrm{HCl})$. The mixture was then allowed to stir for 2 hours under heat. The resultant solution had a moderate viscosity and a transparent or slightly opaque appearance. The viscosity would typically change significantly over the first 24 hours, and the mixture became opaque white overnight. 
Films of a $20 \mathrm{wt} . \%$ PVOH solution were prepared on the QD Proofer and tested to provide an OTR as a reference point. It was also important to verify that the OTRs obtained for these PVOH films matched OTRs given in literature. This would confirm that the method being used to prepare the PVOH coating did, in fact, form a strong oxygen barrier. A second batch of films was prepared using the $20 \mathrm{wt} . \%$ PVOH stock solution with 1 wt.\% Stellar 420 added. This additional experimentation was done to see if addition of a filler material would disrupt the formation of the PVOH resin. The resultant films were around 0.88 microns thick. The OTRs obtained for the $20 \mathrm{wt} . \%$ PVOH film and the $20 \mathrm{wt} . \%$ PVOH film with $1 \mathrm{wt} . \%$ Stellar 420 added, were $2.10 \mathrm{cc} \mathrm{m}^{-2}$ day $^{-1}$ (98.7\% reduction) and $4.61 \mathrm{cc} \mathrm{m}^{-2} \mathrm{day}^{-1}$ (97.0\% reduction) respectively. Primarily, these results confirm that the PVOH coatings produced on the QD Proofer were again within the target thickness of $1-2$ microns and provided OTRs as low as $2 \mathrm{cc} \mathrm{m}^{-2} \mathrm{day}^{-1}$. The second insight taken from the early OTR testing is that the integration of a filler material didn't significantly damage the oxygen barrier properties of the PVOH resin, but clearly didn't shown any reduction in OTR either.

A major concern with existing oxygen barrier coatings that this project strived to address was that, on its own, a PVOH coating has poor resistance to water. Due to the highly polar nature of the $\mathrm{PVOH}$ resin, water vapor molecules in the air can readily solubilize into the coating, acting as a plasticizer that disrupts the resin structure. ${ }^{33}$ To quantify how this plasticization process affects OTR, the $20 \mathrm{wt} . \%$ PVOH coating was also tested at $90 \%$ relative humidity. At this high humidity, the OTR testing of the 20 wt.\% PVOH film provided a significantly higher OTR of $51.57 \mathrm{cc} \mathrm{m}^{-2}$ day $^{-1}(67.0 \%$ 
reduction). The addition of VTMS was hypothesized to influence both the solubility and diffusivity of both oxygen gas and water vapor in the PVOH coating.

Early experimentation with this system focused on finding the ideal relative amounts of PVOH and VTMS to optimize their structural resin interactions. When considering the quantity of VTMS to be integrated into the PVOH stock solution, there are competing goals of having enough VTMS to have a synergic effect with the PVOH resin, yet also limiting any reduction of barrier performance due to VTMS damaging the existing resin structure. The ability for PVOH and VTMS to interact positively was thought to depend on the coating viscosity and curing conditions.

The designation of what composition the weight of 5-20wt.\% VTMS referred to was never clearly specified in the paper. ${ }^{46}$ The weights specified by this range of VTMS composition could refer to the mass of VTMS used in preparation of the coating, expressed as a weight percentage of the total mass of PVOH stock solution and VTMS. Another possibility is that the VTMS weight percentages describe the composition of the dry coating obtained once water and the alcohol byproducts had evaporated off. The first of the two interpretations provides a significantly higher amount of VTMS contribution in the dry film composition.

\subsubsection{4/26 PVOH/VTMS Blends}

Since knowing the upper limit to how much VTMS could be integrated into the PVOH resin was also of interest, the interpretation referring to $5-20 \mathrm{wt} . \%$ VTMS used in preparation of the coating was investigated first. The mass of VTMS required to provide $10 \mathrm{wt} . \%$ VTMS, with the remaining $90 \mathrm{wt} . \%$ from the PVOH stock solution, was calculated before preparation of the coatings. Two blends of this PVOH/VTMS 
composition were prepared, one blend using a 10.78 wt.\% PVOH stock solution, and the second blend using a 16.82 wt.\% PVOH stock solution. If the solids percentage and viscosity of the PVOH stock solution affected the coating composition, then comparison of these two blends was expected to help highlight those differences.

The designation of 74 wt.\% PVOH and 26 wt.\% VTMS, shown in Table 13, refers to the weight percentage in the dry coating composition that was contributed by the initial 90 wt.\% PVOH and 10 wt.\% VTMS quantities, based on theoretical calculations developed after these 74/26 PVOH/VTMS blends. These calculations assume VTMS reacts to completion with itself, yielding a reduced mass of VTMS in the dry coating. The masses used to prepare these coatings and properties such as viscosity and solids percentage of the coating are provided as well.

Table 13. Summary of reagent quantities and resultant properties for the 74/26 PVOH/VTMS blends

\begin{tabular}{|l|c|c|c|c|c|}
\hline $\begin{array}{c}\text { Coating } \\
\text { Composition }\end{array}$ & $\begin{array}{c}\text { Solids } \\
\text { Percent of } \\
\text { PVOH Stock } \\
\text { (wt.\%) }\end{array}$ & $\begin{array}{c}\text { Mass of } \\
\text { PVOH } \\
\text { Stock } \\
\text { (g) }\end{array}$ & $\begin{array}{c}\text { Mass of } \\
\text { VTMS } \\
\text { (g) }\end{array}$ & $\begin{array}{c}\text { Solids Percent } \\
\text { of } \\
\text { PVOH/VTMS } \\
\text { Blend (wt.\%) }\end{array}$ & $\begin{array}{c}\text { Viscosity } \\
\text { at 24 } \\
\text { hours } \\
\text { (mPa.s.) }\end{array}$ \\
\hline $\begin{array}{l}74 \text { wt.\% } \\
\text { PVOH/ 26 } \\
\text { wt.\% VTMS }\end{array}$ & 10.78 & 80.0 & 8.89 & 15.57 & 55 \\
\hline $\begin{array}{l}74 \text { wt.\% } \\
\text { PVOH/26 } \\
\text { wt.\% VTMS }\end{array}$ & 16.82 & 89.8 & 9.98 & 21.98 & 437 \\
\hline
\end{tabular}

The coatings prepared using these formulations were moderately viscous and translucent once left overnight. The solids percentage of PVOH within the stock solution appeared to be a controlling factor influencing the solids percentage and viscosity of the resultant PVOH/VTMS coating. Ideally, the solids of all the PVOH stock solutions would have been maintained at the same percentage. However, evaporation of water during 
preparation of the PVOH stock solutions caused difficulties in ensuring that the expected solids percentage was actually obtained. Both of the coating viscosities changed during the initial 24 hours; from 50 to $55 \mathrm{mPa}$.s. for the 74/26 blend prepared with the 10.78 wt.\% PVOH stock solution, while the 74/26 blend prepared with the $16.82 \mathrm{wt} . \% \mathrm{PVOH}$ stock solution experienced an increase from 338 to $437 \mathrm{mPa}$.s. The trend in these viscosity curves suggests that a high initial viscosity results in a larger increase in viscosity over the first 24 hours. This trend was a promising discovery, since at the lower viscosity of $50 \mathrm{mPa} . \mathrm{s}$, the change in the viscosity was minimal. If this oxygen barrier coating was to become an industrial product, having a working viscosity that doesn't vary significantly over the product's lifetime is a good quality for the coating to have.

Films were prepared on the QD Proofer for the coating variations. Only the 74/26 $\mathrm{PVOH} / \mathrm{VTMS}$ film prepared using the $16.82 \mathrm{wt} . \% \mathrm{PVOH}$ stock solution was tested for oxygen transmission rate. An OTR of $101.11 \mathrm{cc} \mathrm{m}^{-2}$ day $^{-1}$ (35.3\% reduction) suggested that the PVOH/VTMS ratio established here was not optimal, showing poor oxygen barrier performance. Compared to that of the films prepared with a $20 \mathrm{wt} . \% \mathrm{PVOH}$ solution in Table 14, it was believed that the high VTMS content in the 74/26 $\mathrm{PVOH} / \mathrm{VTMS}$ coating reduced the effectiveness of the PVOH resin by disrupting the crystalline resin structure and adding defects to the coating composition that cause a moderate oxygen barrier performance between that of unaltered PVOH films and the bare PET substrate. If significant enough of defects or holes had developed within the coating, the OTR should have approached $156 \mathrm{cc} \mathrm{m}^{-2}$ day $^{-1}$, as observed for the 12 micron PET substrate. 
Table 14. Oxygen transmission rates and thicknesses obtained for the PVOH and 74/26 PVOH/VTMS films

\begin{tabular}{|l|c|c|}
\hline \multicolumn{1}{|c|}{ Coating Composition } & $\begin{array}{c}\text { Coating Thickness } \\
(\boldsymbol{\mu m})\end{array}$ & $\begin{array}{c}\text { OTR at 0\% RH } \\
\left(\mathbf{c c} \mathbf{~ m}^{-\mathbf{2}} \mathbf{d a y}^{-\mathbf{1}} \mathbf{)}\right.\end{array}$ \\
\hline PET- Uncoated & & $156.13 \pm 10.75$ \\
\hline PET- 20 wt.\% PVOH & 0.88 & $2.10 \pm 0.73$ \\
\hline PET- PVOH with 1 wt.\% Stellar 420 & 0.88 & $4.61 \pm 1.17$ \\
\hline PET- 74 wt.\% PVOH/26 wt.\% VTMS & 1.05 & $101.11 \pm 14.28$ \\
\hline
\end{tabular}

A Thermo Fisher Fourier Transform Infrared Spectrometer (FTIR) with ATR 380 was used to track differences in chemical bonding stretches between the PVOH stock solution and the PVOH/VTMS blends. Use of this spectroscopic method could verify the successful condensation reaction between hydroxyl groups from a PVOH chain and a VTMS molecule. Formation of a covalent $\mathrm{Si}-\mathrm{O}-\mathrm{C}$ bond between $\mathrm{PVOH}$ and VTMS was confirmed from comparing the FTIR spectra of VTMS (purple trace) and PVOH/VTMS (red trace) illustrated in Figure 7. Using the stretching associated with the $\mathrm{Si}-\mathrm{O}-\mathrm{CH}_{3}$ linkage, found at a wavenumber of $970 \mathrm{~cm}^{-1}$, it is clear that this peak is present in the VTMS trace, but not as prominently in the PVOH/VTMS trace. The implication of this observation is that the PVOH/VTMS coating contains less of the original alkoxysilane linkages, signifying successful reaction of VTMS with PVOH. Another indication of the success of this reaction is shown by the broadening of the $\mathrm{C}-\mathrm{O}$ peak at $1076 \mathrm{~cm}^{-1}$ in the PVOH/VTMS trace. This occurrence confirms the formation of a Si-O-C cross-linking network between the silanol groups of hydrolyzed VTMS and the pendant hydroxyl groups of PVOH. 


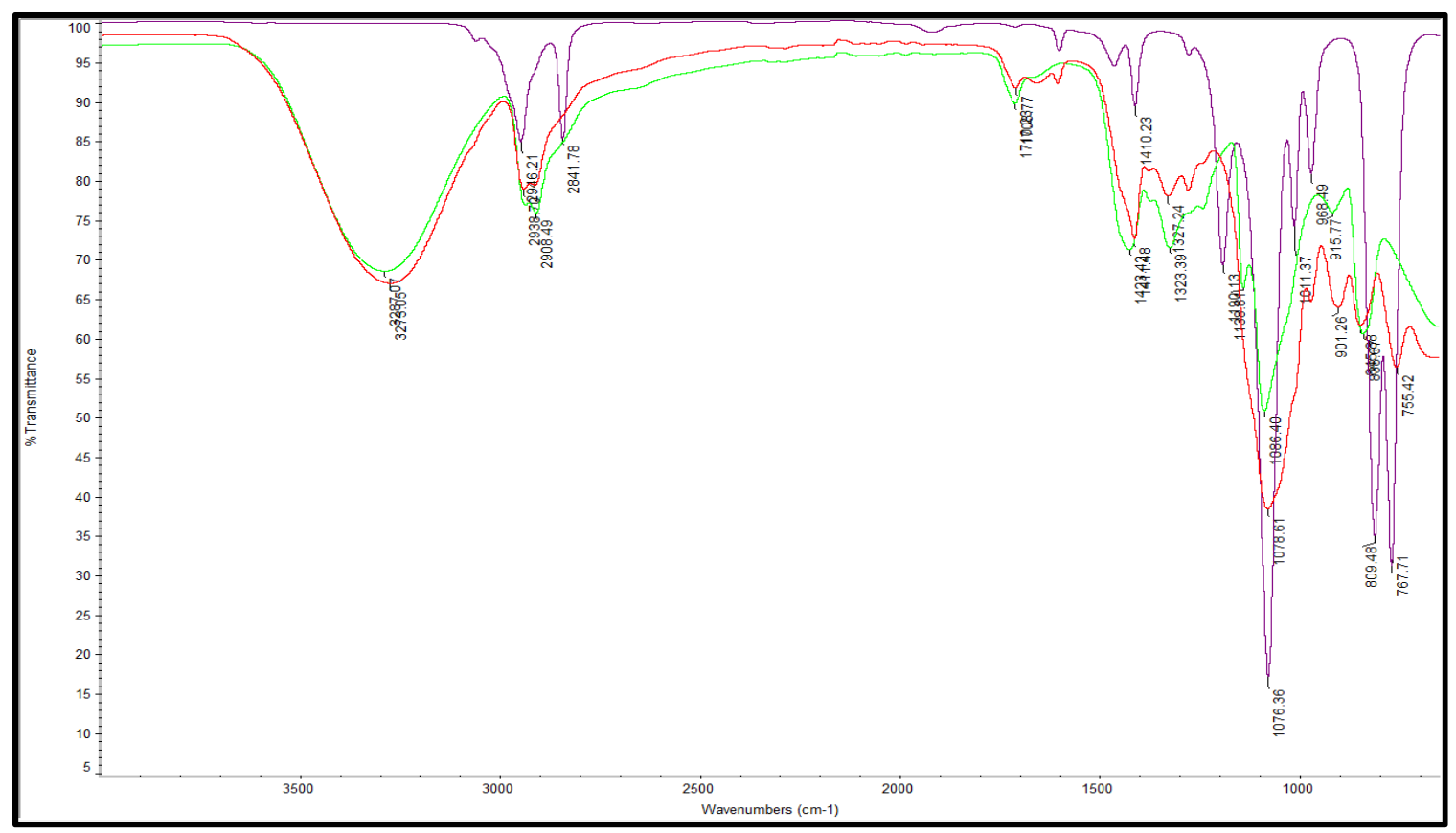

Figure 7. ATR-FTIR overlay of the traces of VTMS (purple), PVOH (green), and 74/26 PVOH/VTMS (red) films

An important observation seen in these 76/24 PVOH/VTMS films during POM, was that consistent ellipsoidal structures were observed throughout the coating layer. The 5x magnification image taken of a subsection of the 74/26 PVOH/VTMS film is shown in Figure 8. Imaging from an unaltered $\mathrm{PVOH}$ film is also provided at 5x magnification as a reference. As depicted, the sheer abundance of these ellipsoidal regions definitely suggested that the resin structure of the PVOH coating is significantly altered by addition of VTMS. The ellipsoidal structures were assumed to be ordered microdomains formed due to the reaction between PVOH and VTMS molecules. Identification of these microdomains was established as a screening method indicating a successful PVOH/VTMS sol-get network. The poor results obtained from the OTR testing and the high frequency of cratering as observed under the POM were taken as indications that the VTMS content was beyond the optimal level in the 74/26 PVOH/VTMS formulations. 


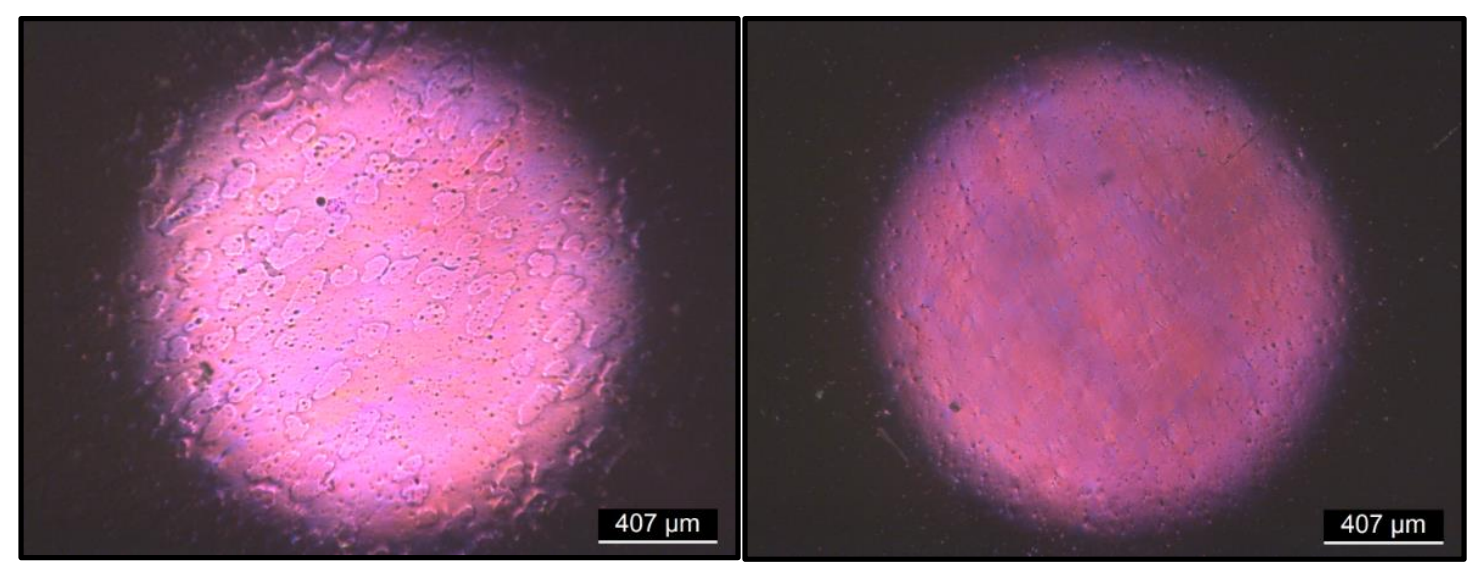

Figure 8. POM image of the 74/26 PVOH/VTMS film (left) compared to an unaltered $\mathrm{PVOH}$ film (right) at $5 \mathrm{x}$ magnification

\subsubsection{5/15 and 97/3 PVOH/VTMS Blends}

The ellipsoidal shapes occurring frequently within the 74/26 PVOH/VTMS films was justification enough to expect that VTMS was added in too high of an amount. A secondary PVOH/VTMS blend was prepared intending a dry coating composition reflecting $90 \mathrm{wt} . \% \mathrm{PVOH}$ and $10 \mathrm{wt} . \%$ VTMS contribution to the resin structure. Another blend was prepared to have $95 \mathrm{wt} . \%$ PVOH and $5 \mathrm{wt}$ \% VTMS contribution. Since the optimal VTMS contribution to the resin structure was expected to be found within the range of $5-20 \mathrm{wt} . \%$ the purpose behind these two PVOH/VTMS blends was to help identify a VTMS amount that provides the highest oxygen barrier performance. Several of the properties obtained through preparation and analysis of these compositions are summarized in Table 15 . The theoretically calculated dry film compositions calculated for these PVOH/VTMS blends were again illustrated through the coating composition designations of 85/15 PVOH/VTMS and 93/7 PVOH/VTMS. 
Table 15. Summary of reagent quantities and resultant properties for the 85/15 and 93/7 PVOH/VTMS blends

\begin{tabular}{|l|c|c|c|c|c|}
\hline $\begin{array}{c}\text { Coating } \\
\text { Composition }\end{array}$ & $\begin{array}{c}\text { Solids } \\
\text { Percent of } \\
\text { PVOH Stock } \\
\text { (wt.\%) }\end{array}$ & $\begin{array}{c}\text { Mass of } \\
\text { PVOH } \\
\text { stock } \\
\text { (g) }\end{array}$ & $\begin{array}{c}\text { Mass of } \\
\text { VTMS } \\
\text { (g) }\end{array}$ & $\begin{array}{c}\text { Solids Percent } \\
\text { of } \\
\text { PVOH/VTMS } \\
\text { Blend (wt.\%) }\end{array}$ & $\begin{array}{c}\text { Viscosity } \\
\text { at 24 } \\
\text { hours } \\
\text { (mPa.s.) }\end{array}$ \\
\hline $\begin{array}{l}85 \text { wt.\% } \\
\text { PVOH/ 15 } \\
\text { wt.\% VTMS }\end{array}$ & 13.22 & 90.0 & 4.00 & 20.79 & 499 \\
\hline $\begin{array}{l}93 \text { wt.\% } \\
\text { PVOH/7 } \\
\text { wt.\% VTMS }\end{array}$ & 15.13 & 39.9 & 0.90 & 15.09 & 165 \\
\hline
\end{tabular}

The oxygen barrier improvement from the 74/26 PVOH/VTMS coating to the newly prepared 85/15 PVOH/VTMS coating was immediately apparent. The 85/15 $\mathrm{PVOH} / \mathrm{VTMS}$ coating provided major oxygen transmission rate reduction of $86 \%$ compared to OTR of the PET substrate. The OTR of the 85/15 PVOH/VTMS film was $22.22 \mathrm{cc} \mathrm{m}^{-2}$ day $^{-1}$ (85.8\% reduction) at $0 \%$ RH. Despite significant improvement, however, this OTR was still an order of magnitude high than the OTR for the $20 \mathrm{wt} . \%$ PVOH film of $2.10 \mathrm{cc} \mathrm{m}^{-2}$ day $^{-1}$. To identify if the VTMS addition helped addressed the problem of water plasticization, the OTR for the 85/15 PVOH/VTMS film was also tested at $90 \% \mathrm{RH}$. The testing provided an OTR of $20.1 \mathrm{cc} \mathrm{m}^{-2}$ day $^{-1}$ (87.1\% reduction) for this high humidity condition. Given the error associated with the $0 \%$ and $90 \%$ OTRs, these values suggest that full retention of oxygen barrier performance was achieved in the presence of high water vapor concentration. The oxygen transmission rates of the 93/7 $\mathrm{PVOH} / \mathrm{VTMS}$ composition were higher, testing yielding OTRs of $64.50 \mathrm{cc} \mathrm{m}^{-2} \mathrm{day}^{-1}$ (58.7\% reduction) at $0 \% \mathrm{RH}$ and $78.11 \mathrm{cc} \mathrm{m}^{-2}$ day $^{-1}$ (50.0\% reduction) at $90 \% \mathrm{RH}$ as shown in Table 16. The OTR of the $20 \mathrm{wt} . \%$ PVOH films obtained at $90 \% \mathrm{RH}$ is provided for comparison. 
Table 16. Oxygen transmission rates and thicknesses obtained for the 85/15, 93/7 single layer, and 93/7 double-layer PVOH/VTMS films

\begin{tabular}{|l|c|c|c|}
\hline \multicolumn{1}{|c|}{ Coating Composition } & $\begin{array}{c}\text { Thickness } \\
(\boldsymbol{\mu m})\end{array}$ & $\begin{array}{c}\text { OTR at 0\% RH } \\
\left(\mathbf{c c ~ m}^{-\mathbf{2}} \mathbf{d a y}^{-1}\right)\end{array}$ & $\begin{array}{c}\text { OTR at 90\% RH } \\
\left(\mathbf{c c ~ m}^{-\mathbf{2}} \mathbf{d a y}^{-1}\right)\end{array}$ \\
\hline PET- 20 wt.\% PVOH & 0.88 & $2.10 \pm 0.73$ & $51.57 \pm 22.74$ \\
\hline $\begin{array}{l}\text { PET- 74 wt.\% PVOH/26 } \\
\text { wt.\% VTMS }\end{array}$ & 1.05 & $101.11 \pm 14.28$ & \\
\hline $\begin{array}{l}\text { PET- 85 wt.\% PVOH/15 } \\
\text { wt.\% VTMS }\end{array}$ & 1.84 & $22.22 \pm 2.93$ & $20.10 \pm 3.68$ \\
\hline $\begin{array}{l}\text { PET- 93 wt.\% PVOH/7 } \\
\text { wt.\% VTMS }\end{array}$ & 0.74 & $64.50 \pm 16.67$ & $78.11 \pm 13.59$ \\
\hline $\begin{array}{l}\text { PET- 93 wt.\% PVOH/7 } \\
\text { wt.\% VTMS (Double-layer) }\end{array}$ & 1.81 & $19.67 \pm 8.17$ & \\
\hline
\end{tabular}

The thickness of the 93/7 PVOH/VTMS films were found to be considerably thinner than the 74/26 and 85/15 PVOH/VTMS films with an average thickness of 0.74 microns. This thin coating was considered to be caused by the low solids and viscosity of the $93 / 7$ PVOH/VTMS coating. To obtain thicker films, double-layer films were prepared on the QD Proofer using the 93/7 PVOH/VTMS blend. The typical film-making process was changed to include a second QD Proof film deposition, occurring once the first layer had been dried and cured at $60^{\circ} \mathrm{C}$ for one hour. The double-layer film was then cured for an additional one hour at the same temperature.

This film preparation technique increased the single layer 93/7 PVOH/VTMS coating thickness of 0.74 microns to 1.81 microns through addition of a second layer. This thickness closely resembles the 1.84 micron thickness obtained from the 93/7 $\mathrm{PVOH} / \mathrm{VTMS}$ coatings allowing these compositions to be compared at the same coating thickness. An OTR of $19.67 \mathrm{cc} \mathrm{m}^{-2} \mathrm{day}^{-1}$ (87.4\% reduction) at $0 \% \mathrm{RH}$ was measured for these double-layer 93/7 PVOH/VTMS films compared to an OTR of $22.22 \mathrm{cc} \mathrm{m}^{-2} \mathrm{day}^{-1}$ (85.8\% reduction) for the 85/15 single PVOH/VTMS layer films. Since the thicknesses of 
these two PVOH/VTMS films were the same, the consistent oxygen barrier performance between the $7 \%$ and $15 \%$ VTMS compositions suggests that the VTMS contribution to the resin structure in these two blends is similar. Unfortunately the 90\% RH OTR for the 93/7 PVOH/VTMS double-layer films was never measured since only one film was prepared. Despite this observation, having a single layer of the 85/15 PVOH/VTMS film provides a simpler processing route, which is a significant consideration since these coatings were going to be scaled up on an industrial printing press.

Images of the 85/15 PVOH/VTMS films were taken under the polarized optical microscope as shown in Figure 9. The ellipsoidal microdomains were observed again, however, the frequency of these shapes was significantly less than in the 74/26 $\mathrm{PVOH} / \mathrm{VTMS}$ films. The actual size of these regions was also smaller in the $85 / 15$ $\mathrm{PVOH} / \mathrm{VTMS}$ films, supporting the claim that they are formed due to VTMS reacting with PVOH to form a unique sol-gel network. The difference in color between the images taken was an effect caused by the settings on the POM and should not be a part of any comparisons between the films.

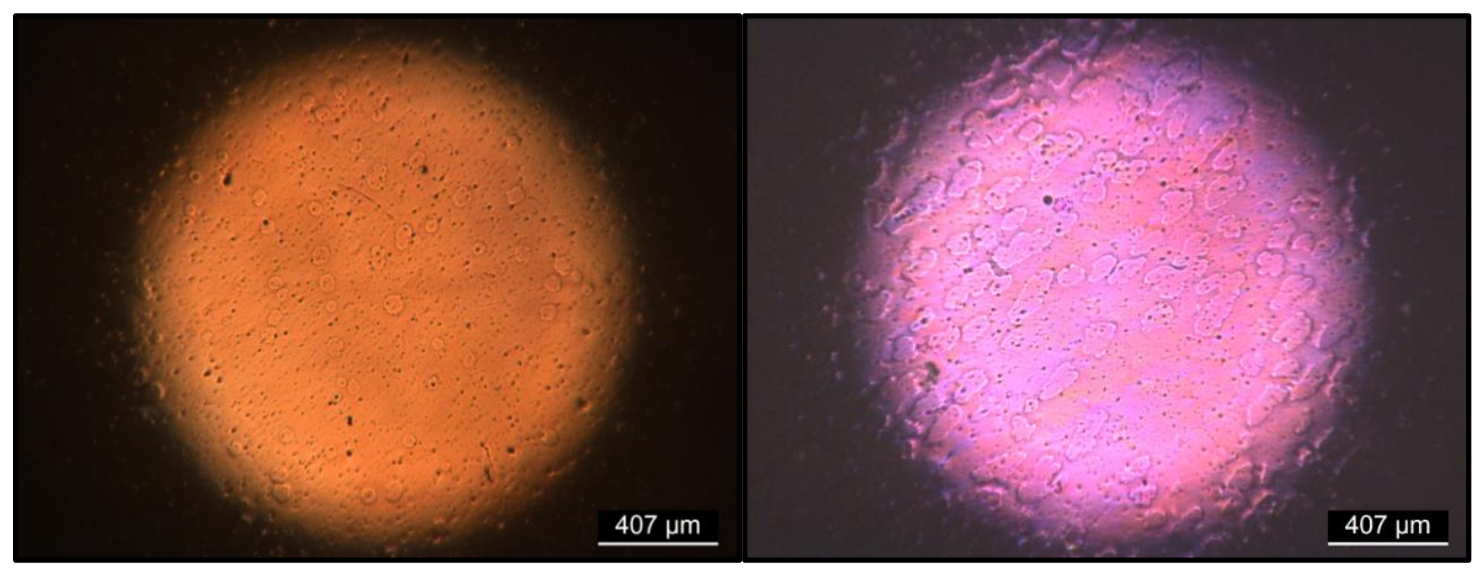

Figure 9. POM images of the $85 / 15$ (left) and 74/26 (right) PVOH films at 5x magnification 


\subsubsection{0/10 PVOH/VTMS Blends}

The composition of the PVOH/VTMS dry coatings was re-evaluated again to taking into account the theoretical chemical reactions occurring between these molecules from the understanding of the sol-gel chemistry involved. In this interpretation, molar ratios related to the hydrolysis and condensation reactions, were used to predict the masses of PVOH and VTMS that are integrated into the resin structure. Essentially, the loss of water and alcohols as byproducts results in a lower mass of both PVOH and VTMS, in the dry coating, than was initially added. The prior designations of $74 / 26,85 / 15$, and 93/7 PVOH/VTMS for the previous blends are calculated from these predictions.

Using this new modelling, calculations were done to prepare a dry coating composition that was actually from $90 \mathrm{wt} . \%$ PVOH and $10 \mathrm{wt} . \%$ VTMS contribution. The 90/10 PVOH/VTMS formulation used for this composition is provided in Table 17. Films of the 90/10 PVOH/VTMS coating composition were prepared on the QD Proofer. Surprisingly, despite a higher viscosity and solids percentage, the 90/10 PVOH/VTMS blend provided a thinner coating layer that was 1.59 microns thick compared to the 1.84 micron thick coating layer from the 85/5 PVOH/VTMS composition. These 90/10 PVOH/VTMS films were observed under the POM and the occurrence of ellipsoidal structures was similar to those seen in the 85/15 PVOH/VTMS films. 
Table 17. Summary of properties and reagent quantities for the 90/10 PVOH/VTMS blend

\begin{tabular}{|l|c|c|c|c|c|}
\hline $\begin{array}{c}\text { Coating } \\
\text { Composition }\end{array}$ & $\begin{array}{c}\text { Solids } \\
\text { Percent of } \\
\text { PVOH } \\
\text { Stock } \\
\text { (wt.\%) }\end{array}$ & $\begin{array}{c}\text { Mass of } \\
\text { PVOH } \\
\text { stock } \\
\text { (g) }\end{array}$ & $\begin{array}{c}\text { Mass of } \\
\text { VTMS } \\
\text { (g) }\end{array}$ & $\begin{array}{c}\text { Solids Percent } \\
\text { of PVOH/ } \\
\text { VTMS Blend } \\
\text { (wt.\%) }\end{array}$ & $\begin{array}{c}\text { Viscosity at } \\
\text { 24 hours } \\
\text { (mPa.s.) }\end{array}$ \\
\hline $\begin{array}{l}\text { 90 wt.\% } \\
\text { PVOH/ } 10 \\
\text { wt.\% VTMS }\end{array}$ & 18.90 & 90.0 & 3.55 & 20.74 & 905 \\
\hline
\end{tabular}

Despite producing thinner coatings compared to the 85/15 PVOH/VTMS coating, OTRs of $6.87 \mathrm{cc} \mathrm{m}^{-2} \mathrm{day}^{-1}$ (95.6\% reduction) at $0 \% \mathrm{RH}$ and $16.13 \mathrm{cc} \mathrm{m}^{-2} \mathrm{day}^{-1}(89.7 \%$ reduction) at 90\% RH were obtained for the 90/10 PVOH/VTMS films (Table 18). These low oxygen transmission rates approach the target performance expected for these oxygen barrier coatings. The near full retention of oxygen barrier performance when the OTR testing was done under high humidity conditions of $90 \% \mathrm{RH}$ on these $\mathrm{PVOH} / \mathrm{VTMS}$ coatings is also significant, justifying the slight increase in OTR at $0 \% \mathrm{RH}$ when compared to pure PVOH coatings.

Table 18. Oxygen transmission rates and thicknesses obtained for the 90/10 PVOH/VTMS films

\begin{tabular}{|c|c|c|c|}
\hline Coating Composition & $\begin{array}{c}\text { Thickness } \\
(\mu \mathrm{m})\end{array}$ & $\begin{array}{c}\text { OTR at 0\% RH } \\
\left(\text { cc m }^{-2} \text { day }^{-1}\right)\end{array}$ & $\begin{array}{c}\text { OTR at 90\% RH } \\
\left(\text { cc m }^{-2} \text { day }^{-1}\right)\end{array}$ \\
\hline PET- 20 wt. $\%$ PVOH & 0.88 & $2.10 \pm 0.73$ & $51.57 \pm 22.74$ \\
\hline $\begin{array}{l}\text { PET- } 85 \text { wt. } \% \text { PVOH/15 } \\
\text { wt.\% VTMS }\end{array}$ & 1.84 & $22.22 \pm 2.93$ & $20.10 \pm 3.68$ \\
\hline $\begin{array}{l}\text { PET- } 90 \text { wt. } \% \text { PVOH/10 } \\
\text { wt.\% VTMS }\end{array}$ & 1.59 & $6.87 \pm 2.53$ & $16.13 \pm 1.17$ \\
\hline
\end{tabular}


The 90/10 PVOH/VTMS films demonstrated two of the main goals of the project: excellent oxygen barrier performance under standard conditions, and retention of this barrier ability when films were exposed to high humidity. Experimentation was next shifted to adapt the 90/10 PVOH/VTMS coating properties to be optimal for use under scaled up press printing conditions. A major concern with the existing state of the 90/10 PVOH coating was the high viscosity of $905 \mathrm{mPa}$.s. Ideally, the working viscosity of this coating would be below $100 \mathrm{mPa}$.s. Even a viscosity below $300 \mathrm{mPa}$.s was considered to meet requirements for industrial press use.

\subsubsection{Diluted 90/10 PVOH/VTMS Blends}

Drawing knowledge from previous coating preparation, a strong correlation had been observed between the solids percentage in the PVOH/VTMS coating and its viscosity. The solids of the 90/10 PVOH/VTMS coating was $20.74 \mathrm{wt} . \%$, which is the highest of any of the PVOH/VTMS blends prepared. Consequently, the high viscosity of the 90/10 PVOH/VTMS coating should be expected. The easiest way to prepare a 90/10 $\mathrm{PVOH} / \mathrm{VTMS}$ coating with lower viscosity was to dilute the coating by adding deionized water in the coating formulation. By adding water to the 90/10 PVOH/VTMS formulation, the solids percentage of the resultant coating is reduced, while still maintaining the dry coating composition.

This concept was put into practice by preparing two diluted 90/10 PVOH/VTMS coatings where deionized water was added in moderate portions. The formulations of these diluted 90/10 PVOH/VTMS variations are summarized in Table 19. The resultant moderately diluted and highly diluted 90/10 PVOH/VTMS coatings had 24 hour viscosities of 275 and $100 \mathrm{mPa}$.s respectively, corresponding to solids of $18.46 \mathrm{wt} . \%$ and 
$14.69 \mathrm{wt} . \%$. These two blends established a range of target solids percentages for these 90/10 PVOH/VTMS coatings that should provide viscosities around $100-275 \mathrm{mPa} . \mathrm{s}$.

Films were only prepared with the moderate viscosity 90/10 PVOH/VTMS blend in double-layer fashion, resulting in an average coating thickness of 1.22 microns. The goal of including a second layer in the film preparation of this moderate viscosity 90/10 $\mathrm{PVOH} / \mathrm{VTMS}$ blend was to maintain the approximate thickness previously obtained with the undiluted PVOH/VTMS coatings, while correcting any surface defects that could result from the thinner coating.

Table 19. Summary of properties and reagent quantities for the diluted 90/10 PVOH/VTMS blends

\begin{tabular}{|c|c|c|c|c|c|c|}
\hline $\begin{array}{c}\text { Coating } \\
\text { Composition }\end{array}$ & $\begin{array}{c}\text { Solids } \\
\text { Percent of } \\
\text { PVOH } \\
\text { Stock } \\
\text { (wt. \%) }\end{array}$ & $\begin{array}{c}\text { Mass of } \\
\text { PVOH } \\
\text { stock } \\
\text { (g) }\end{array}$ & $\begin{array}{l}\text { Mass } \\
\text { of } \\
\text { VTMS } \\
(\mathrm{g})\end{array}$ & $\begin{array}{c}\text { Mass } \\
\text { of DI } \\
\text { Water } \\
\text { (g) }\end{array}$ & $\begin{array}{c}\text { Solids } \\
\text { Percent of } \\
\text { PVOH/ } \\
\text { VTMS } \\
\text { Blend } \\
\text { (wt.\%) }\end{array}$ & $\begin{array}{c}\text { Viscosity } \\
\text { at } 24 \\
\text { hours } \\
\text { (mPa.s) }\end{array}$ \\
\hline $\begin{array}{l}\text { Moderate } \\
\text { Dilution 90 } \\
\text { wt.\% PVOH/ } \\
10 \text { wt.\% } \\
\text { VTMS }\end{array}$ & 17.91 & 66.3 & 2.47 & 11.2 & 18.46 & 275 \\
\hline $\begin{array}{l}\text { High Dilution } \\
90 \text { wt.\% } \\
\text { PVOH/10 } \\
\text { wt.\% VTMS }\end{array}$ & 19.56 & 55.4 & 2.24 & 22.6 & 14.69 & 100 \\
\hline
\end{tabular}

The oxygen transmission rate testing of this moderately diluted 90/10 PVOH/VTMS composition helped confirm that the same performance, as seen in the 90/10 PVOH/VTMS coating, was achievable despite the reduction in viscosity and solids content of these diluted coatings. These double-layer films yielded OTRs of $7.05 \mathrm{cc} \mathrm{m}^{-2}$ day $^{-1}\left(95.5 \%\right.$ reduction) at $0 \% \mathrm{RH}$ and $15.68 \mathrm{cc} \mathrm{m}^{-2}$ day $^{-1}(90.0 \%$ reduction) at $90 \% \mathrm{RH}$ 
as listed in Table 20. These OTRs are comparable to the barrier performance obtained with the undiluted 90/10 PVOH/VTMS films. At this point, the PVOH/VTMS coatings were considered to be developed enough for use in scaled up film preparation using an industrial printing press.

Table 20. Oxygen transmission rates and thicknesses obtained for the diluted 90/10 PVOH/VTMS films

\begin{tabular}{|l|c|c|c|}
\hline \multicolumn{1}{|c|}{ Coating Composition } & $\begin{array}{c}\text { Thickness } \\
(\boldsymbol{\mu m})\end{array}$ & $\begin{array}{c}\text { OTR at 0\% RH } \\
\left(\mathbf{c c ~ m}^{-\mathbf{2}} \mathbf{d a y}^{-\mathbf{1}}\right)\end{array}$ & $\begin{array}{c}\text { OTR at 90\% RH } \\
\left(\mathbf{c c ~ m}^{-2} \mathbf{d a y}^{-1}\right)\end{array}$ \\
\hline PET- 20 wt.\% PVOH & 0.88 & $2.10 \pm 0.73$ & $51.57 \pm 22.74$ \\
\hline $\begin{array}{l}\text { PET- 90 wt.\% PVOH/10 } \\
\text { wt.\% VTMS }\end{array}$ & 1.59 & $6.87 \pm 2.53$ & $16.13 \pm 1.17$ \\
\hline $\begin{array}{l}\text { PET- Diluted 90 wt.\% } \\
\text { PVOH/10 wt.\% VTMS }\end{array}$ & 1.22 & $7.05 \pm 5.30$ & $15.86 \pm 3.60$ \\
\hline
\end{tabular}

\subsubsection{Block Testing of 90/10 PVOH/VTMS Films}

A standard ink-to-substrate block resistance test was conducted with these diluted 90/10 PVOH/VTMS coatings, under ambient and heated conditions, to test if films could be stored wrapped up on a spool. The expectation was that these films could be directly rolled onto a spool after being run through the press without causing damage to the coating's integrity. A diluted 90/10 PVOH/VTMS coating was freshly formulated with a viscosity of $\sim 100 \mathrm{mPa}$.s and single-layer films were prepared on the QD Proofer. After deposition, these films were immediately dried using a heated blow drier for a minute. Two film sections were folded upon themselves so that segments of the coating were in contact with uncoated PET substrate. One of these sections was left on a shelf in ambient conditions, while the other was placed into the oven for 18 hours, heated to $50^{\circ} \mathrm{C}$. A paint can, filled with ten pounds of lead shot, was placed on top of each folded samples, with a 1 " $\mathrm{x} 1$ " rubber piece in between. This setup was meant to simulate $10 \mathrm{psi}$ of pressure on 
the rolled films sections and was used to determine whether any sticking or blocking would occur under these conditions. The film samples were unfolded after 18 hours and were inspected to see if any blocking occurred in either ambient or heated conditions. In both cases, no blocking was observed when unfolding the film. These tests verified that spools could be used to store the sheets of coated films, without concern over the integrity of the coating.

Prior to conducting the trial press printing, several aspects of the synthetic procedure were investigated to address concerns such as environmental effects and safety precautions, related to the materials and by-products involved with these PVOH/VTMS coatings. These aspects were not of immediate concern but were expected to improve the PVOH/VTMS coating preparation process. In industry, the use of strong acids, hydrochloric acid or sulfuric acid $\left(\mathrm{H}_{2} \mathrm{SO}_{4}\right)$, is avoided due to their extreme corrosive nature. Since the level of acidity necessary to catalyze sol-gel reactions isn't well defined, a weaker acid such as citric acid $\left(\mathrm{C}_{6} \mathrm{H}_{8} \mathrm{O}_{7}\right)$ was proposed as an alternative to concentrated hydrochloric acid. A second issue is that methanol is produced as a by-product during the hydrolysis step of the PVOH/VTMS reaction. Use of triethoxyvinylsilane or tetraethylorthosilane would instead generate ethanol during these sol-gel reactions. Even though these solvents evaporate during film formation, having ethanol in residual amounts would be more desirable from an industrial production standpoint than methanol. Methanol is widely considered to be a toxic solvent in commercial industry. Finally, the need for elevated temperatures both within the preparation itself and also to force cure the film samples was also questioned. Integration of heating stages in the film preparation method can be both difficult and costly. 


\subsubsection{0/10 PVOH/TEVS and 90/10 PVOH/TEOS Blends}

Two alternate coatings using TEVS or TEOS, in place of VTMS, were prepared using the exact same procedure as the PVOH/VTMS blends. Both of these coatings were formulated to provide $90 \% \mathrm{PVOH}$ and $10 \%$ TEVS or TEOS contribution to the dry coating composition. The specific reagent amounts for these synthetic trials and resultant coating properties are given in Table 21.

Table 21. Summary of properties and reagent quantities for the 90/10 PVOH/TEVS and 90/10 PVOH/TEOS blends

\begin{tabular}{|l|c|c|c|c|c|c|}
\hline $\begin{array}{c}\text { Coating } \\
\text { Composition }\end{array}$ & $\begin{array}{c}\text { Solids } \\
\text { Percent } \\
\text { of PVOH } \\
\text { Stock } \\
\text { (wt.\%) }\end{array}$ & $\begin{array}{c}\text { Mass of } \\
\text { PVOH } \\
\text { stock (g) }\end{array}$ & $\begin{array}{c}\text { Mass of } \\
\text { Cross- } \\
\text { linker } \\
\text { (g) }\end{array}$ & $\begin{array}{c}\text { Mass } \\
\text { of DI } \\
\mathbf{H}_{2} \text { O } \\
\text { (g) }\end{array}$ & $\begin{array}{c}\text { Solids } \\
\text { Percent of } \\
\text { PVOH } \\
\text { Blend } \\
\text { (wt.\%) }\end{array}$ & $\begin{array}{c}\text { Viscosity } \\
\text { at 24 } \\
\text { hours } \\
\text { (mPa.s) }\end{array}$ \\
\hline $\begin{array}{l}\text { 90 wt.\% } \\
\text { PVOH/ 10 } \\
\text { wt.\% TEVS }\end{array}$ & 22.28 & 39.99 & 2.36 & 17.65 & 14.15 & 86 \\
\hline $\begin{array}{l}\text { 90 wt.\% } \\
\text { PVOH/ } 10 \\
\text { wt.\% TEOS }\end{array}$ & 22.28 & 39.99 & 2.51 & 17.5 & 14.59 & 103 \\
\hline
\end{tabular}

There were signs from the preparation and analysis of the films that suggested that neither of these compositions would have as strong an oxygen barrier performance as PVOH/VTMS blends. The first indication that the PVOH/TEVS and PVOH/TEOS coatings were not reacting in the same manner as the PVOH/VTMS mixture was that after 24 hours these mixtures were still transparent. Previously, it was mentioned that a distinct change in the appearance of the PVOH/VTMS mixture occurred overnight, changing from transparent to an opaque white. Weeks after being prepared, the $\mathrm{PVOH} / \mathrm{TEVS}$ and $\mathrm{PVOH} / \mathrm{TEOS}$ mixtures were still transparent suggesting that no 
reaction had proceeded. This was particularly surprising for the PVOH/TEVS composition, since the final chemical composition should be identical to that of the PVOH/VTMS composition. Images taken under the polarized optical microscope of the 90/10 PVOH/TEVS films also provided evidence against a successful reaction between the PVOH and TEVS materials. Ellipsoidal shapes were observed in the 90/10 PVOH/TEOS films, but not in the 90/10 PVOH/TEVS films (Figure 10). Given the frequency of the ellipsoidal shapes in the 90/10 PVOH/TEOS films, this may suggest that the contribution from the TEOS composition is too high, as seen with the 74/26 PVOH/VTMS blend.
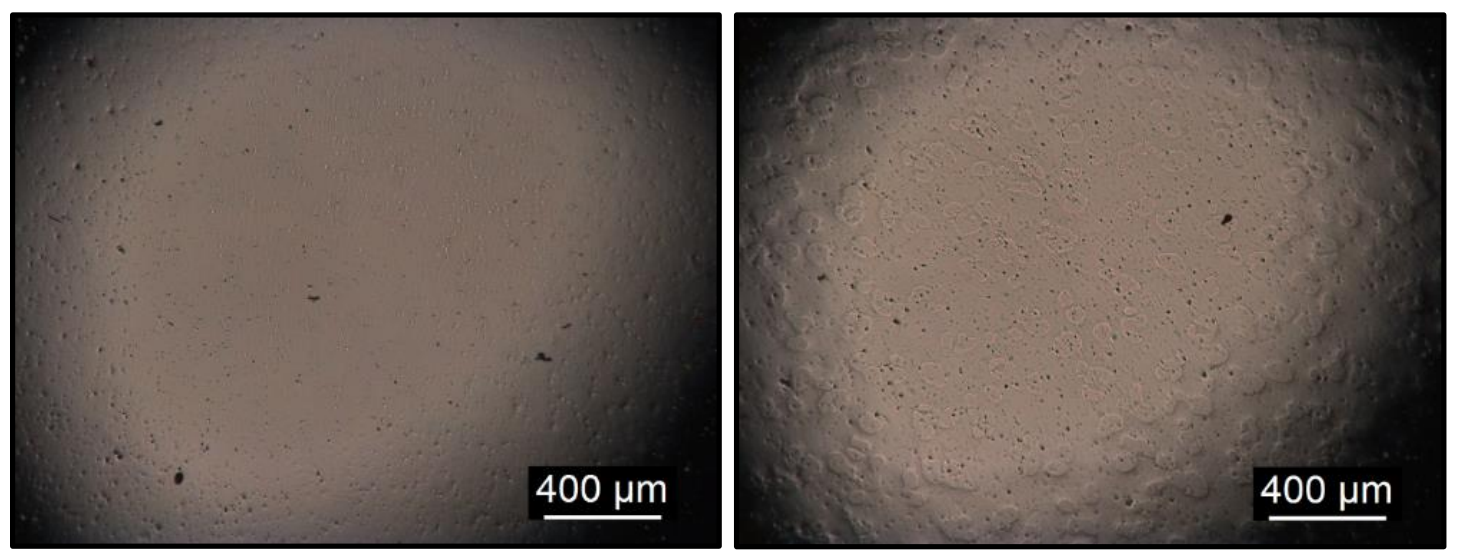

Figure 10. POM images of the 90/10 PVOH/TEVS film (left) and 90/10 PVOH/TEOS film (right) at 5x magnification

The suspicions that arose during analysis of the films were confirmed by OTR testing. Oxygen transmission rates of 96.69 and $94.05 \mathrm{cc} \mathrm{m}^{-2}$ day $^{-1}(38.1 \%$ and $39.8 \%$ reduction) were acquired at $0 \% \mathrm{RH}$ and $90 \% \mathrm{RH}$, as shown in Table 22 , for the $90 / 10$ PVOH/TEVS films . The PVOH/TEOS films had stronger oxygen barrier performance at $0 \%$ RH with an OTR of $61.63 \mathrm{cc} \mathrm{m}^{-2} \mathrm{day}^{-1}$ (60.5\% reduction). These results were not nearly as good as the barrier provided by the PVOH/VTMS coatings. Given the 
similarities in the sol-gel reactions taken place in all of these systems, it was expected that, by varying experimental conditions involved in film preparation, that these compositions could produce strong oxygen barrier coatings. The main conditions that would be changed to prepare successful PVOH/TEOS and PVOH/TEVS oxygen barrier coatings would be the weight percentages of TEOS/TEVS and the temperature used during preparation. For the PVOH/TEOS composition, in particular, the fact that TEOS has four reactive hydroxyl groups once hydrolyzed is an important consideration.

Table 22. Oxygen transmission rates and thicknesses obtained for the 90/10 PVOH/TEVS and 90/10 PVOH/TEOS films

\begin{tabular}{|c|c|c|c|}
\hline Coating Composition & $\begin{array}{l}\text { Thickness } \\
(\mu \mathrm{m})\end{array}$ & $\begin{array}{l}\text { OTR at 0\% RH } \\
\left(\text { (cc } \text { m }^{-2} \text { day }^{-1}\right)\end{array}$ & $\begin{array}{l}\text { OTR at } 90 \% \text { RH } \\
\left(\text { cc m }^{-2} \text { day }^{-1}\right)\end{array}$ \\
\hline PET- 20 wt.\% PVOH & 0.88 & $2.10 \pm 0.73$ & $51.57 \pm 22.74$ \\
\hline $\begin{array}{l}\text { PET- Diluted } 90 \text { wt. } \% \\
\text { PVOH/10 wt.\% VTMS }\end{array}$ & 1.22 & $7.05 \pm 5.30$ & $15.86 \pm 3.60$ \\
\hline $\begin{array}{l}\text { PET- } 90 \text { wt. } \% \text { PVOH/10 } \\
\text { wt. } \% \text { TEOS }\end{array}$ & 1.14 & 96.69 & $94.05 \pm 17.61$ \\
\hline $\begin{array}{l}\text { PET- } 90 \text { wt. } \% \text { PVOH/10 } \\
\text { wt.\% TEVS }\end{array}$ & 1.13 & $61.63 \pm 1.03$ & \\
\hline
\end{tabular}

\subsubsection{0/10 PVOH/VTMS Blend Prepared without Heat}

A 90/10 PVOH/VTMS coating was prepared at $23^{\circ} \mathrm{C}$ instead of the usual $50^{\circ} \mathrm{C}$ to see if the reaction between PVOH and VTMS molecules would still occur. Similar to the $\mathrm{PVOH} / \mathrm{TEVS}$ and PVOH/TEOS coatings, a change in mixture appearance to an opaque white wasn't observed after a 24 hours. Films were prepared using the unheated 90/10 PVOH blend the following day, and an OTR of $71.41 \mathrm{cc} \mathrm{m}^{-2}$ day $^{-1}$ (54.3\% reduction) at $0 \% \mathrm{RH}$ was obtained from testing of these films. After several days, the mixture appearance eventually transitioned from a transparent to opaque nature. This observation 
suggests that applied heat significantly increases rate of the PVOH/VTMS reaction, yet the reaction can still take place slowly over time at ambient conditions. An interesting follow up experiment involving these 90/10 PVOH/VTMS blends prepared without heat would be to re-make films once the mixture had turned opaque and see if the OTR of those newly made films was comparable to heated 90/10 PVOH/VTMS blends. That would clarify if the reaction proceeds to the same final conversion even without heat.

\subsubsection{Stability of $\mathrm{HCl}$ in a PVOH Stock Solution}

The stability of a heated mixture of $\mathrm{PVOH}$ and concentrated $\mathrm{HCl}$ was monitored by ${ }^{1} \mathrm{H}$ NMR using a $400 \mathrm{MHz}$ NMR in the Cal Poly Chemistry and Biochemistry department. This experiment was conducted to confirm whether the PVOH stock solution and $\mathrm{HCl}$ catalyst components of the PVOH/VTMS coating would remain stable when combined. A PVOH/HCl mixture was heated at $50^{\circ} \mathrm{C}$ and let stir for three hours as typical done when preparing $\mathrm{PVOH}$ stock solutions. An aliquot of this $\mathrm{PVOH} / \mathrm{HCl}$ mixture was diluted in deuterated water in an NMR tube. An overlay comparing the NMR spectra of the unreacted PVOH, outlined in blue, and the acidic mixture, outlined in crimson, is shown in Figure 11. The significant peaks for both NMR spectra exactly matched between the two traces, and the relative intensities of the peaks remained the same. The peak at $1.55 \mathrm{ppm}$ is attributed to the hydrogens attached to the alkyl $\left(\mathrm{CH}_{2}\right)$ group along the PVOH backbone, while the 3.90 ppm stretch results from the alkyl $(\mathrm{CH})$ group directly bonded to the pendant hydroxyl group. The remaining peaks are attributed to the deuterated water used to dilute the sample, or the small percentage of unhydrolyzed PVOH within the Poval 4-98 as provided. From this short analysis, it was determined that 
the PVOH and acid catalyst can be combined without reacting, even in the presence of heat due to the lack of signal integration changes between the two spectra.

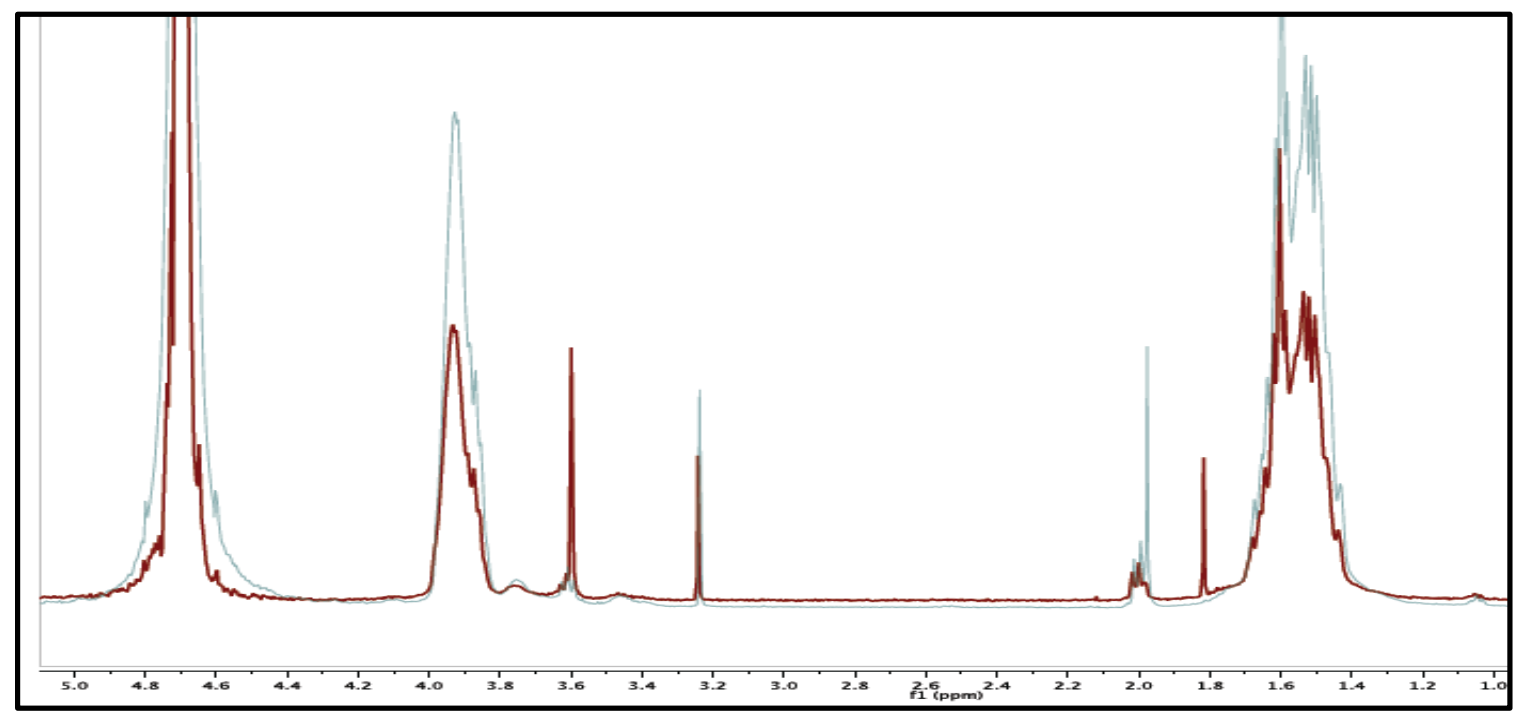

Figure 11. ${ }^{1} \mathrm{H}$ NMR overlay of traces for unreacted $\mathrm{PVOH}$ (blue) and the $\mathrm{PVOH} / \mathrm{HCl}$ mixture (crimson)

\subsubsection{0/10 PVOH/VTMS Blend Prepared with Citric Acid}

The use of citric acid instead of $\mathrm{HCl}$ as a catalyst provided the most success from the alternative preparation routes attempted. Since citric acid is a solid at room temperature, molar masses were used to calculate the equivalent amount of citric acid necessary to provide the same number of moles as found in two drops of $\mathrm{HCl}$. No problems were encountered dissolving the citric acid in the PVOH/VTMS solution and the resultant properties from this coating are listed in Table 23. Double-layer films were prepared in order to attain thicker coatings due to the lower viscosity of this blend. 
Table 23. Summary of properties and reagent quantities for the 90/10 PVOH/VTMS blend prepared with a citric acid catalyst

\begin{tabular}{|l|c|c|c|c|c|c|}
\hline $\begin{array}{c}\text { Coating } \\
\text { Composition }\end{array}$ & $\begin{array}{c}\text { Solids } \\
\text { Percent } \\
\text { of PVOH } \\
\text { Stock } \\
\text { (wt.\%) }\end{array}$ & $\begin{array}{c}\text { Mass } \\
\text { of } \\
\text { PVOH } \\
\text { stock } \\
\text { (g) }\end{array}$ & $\begin{array}{c}\text { Mass } \\
\text { of } \\
\text { VTMS } \\
\text { (g) }\end{array}$ & $\begin{array}{c}\text { Mass } \\
\text { of DI } \\
\text { Water } \\
\text { (g) }\end{array}$ & $\begin{array}{c}\text { Solids } \\
\text { Percent } \\
\text { of PVOH/ } \\
\text { VTMS } \\
\text { Blend } \\
\text { (wt.\%) }\end{array}$ & $\begin{array}{c}\text { Viscosity } \\
\text { at 24 } \\
\text { hours } \\
\text { (mPa.s) }\end{array}$ \\
\hline $\begin{array}{l}90 \text { wt.\% } \\
\text { PVOH/ 10 } \\
\text { wt.\% PVOH } \\
\text { using Citric } \\
\text { Acid Catalyst }\end{array}$ & 22.28 & 39.99 & 1.85 & 18.16 & 16.25 & 133 \\
\hline
\end{tabular}

The 90/10 PVOH/VTMS coating prepared with citric acid showed an improved

OTR, compared to any of the previous compositions, despite thin double-layer films of 0.81 microns and moderate solids content of $16.25 \mathrm{wt} . \%$. The OTR at $0 \% \mathrm{RH}$ was 1.44 $\mathrm{cc} \mathrm{m}^{-2} \operatorname{day}^{-1}$ (99.1\% reduction) and $13.03 \mathrm{cc} \mathrm{m}^{-2}$ day $^{-1}$ (91.7\% reduction) at $90 \% \mathrm{RH}$ (Table 24). A hypothesis to why this weak acid would improve the oxygen barrier performance, when compared to the strong acid variation, was that the citric acid could crosslink with the existing resin, to a small degree. This hypothesis is supported by literature that has used citric acid in low weight percentages as a crosslinking agent, and demonstrated that its inclusion into the resin structure could also improve water resistance. ${ }^{47}$ The testing of these films took place after the trial press run was conducted, otherwise this catalyst replacement may have been used while preparing the 90/10 PVOH/VTMS coating utilized on the press. 
Table 24. Oxygen transmission rates and thicknesses obtained for the 90/10 PVOH/VTMS films prepared with a citric acid catalyst

\begin{tabular}{|c|c|c|c|}
\hline Coating Composition & $\begin{array}{l}\text { Thickness } \\
(\mu \mathrm{m})\end{array}$ & $\begin{array}{l}\text { OTR at 0\% RH } \\
\left(\text { cc } \text { m }^{-2} \text { day }^{-1}\right)\end{array}$ & $\begin{array}{l}\text { OTR at } 90 \% \text { RH } \\
\left(\text { cc }^{-2} \text { day }^{-1}\right)\end{array}$ \\
\hline PET- 20 wt. $\%$ PVOH & 0.88 & $2.10 \pm 0.73$ & $51.57 \pm 22.74$ \\
\hline $\begin{array}{l}\text { PET- Diluted } 90 \text { wt. } \% \\
\text { PVOH/10 wt. } \% \text { VTMS }\end{array}$ & 1.22 & $7.05 \pm 5.30$ & $15.86 \pm 3.60$ \\
\hline $\begin{array}{l}\text { PET- } 90 \text { wt.\% PVOH/ } 10 \\
\text { wt.\% PVOH using Citric } \\
\text { Acid Catalyst }\end{array}$ & 0.81 & $1.44 \pm 0.50$ & $13.35 \pm 0.21$ \\
\hline
\end{tabular}

\subsection{Press Trials}

The setup for this scaled up 90/10 PVOH/VMTS coating preparation was essentially a larger version of the regular procedure. The basic setup included use of a $5 \mathrm{~L}$ round bottom flask with a heating apparatus, an automatic stirrer with a large paddle, and a condenser to prevent evaporation of water. The quantity of Poval 4-98 used in this scaled up formulation turned the solution color to a strong yellow hue as shown in Figure 12. The resultant properties of this scaled up blend are provided in Table 25. The final reaction mixture was transferred into three $1 \mathrm{~L}$ bottles to be transported and used in the press trial. The larger reaction setup allowed for better control of heating, as well as higher precision in obtaining the desired solids percentage from the resultant coating than possible in coating preparation on the smaller scale. 
Table 25. Summary of properties and reagent quantities for the 90/10 PVOH/VTMS blend used for scaled up press run

\begin{tabular}{|l|c|c|c|c|c|c|}
\hline $\begin{array}{c}\text { Coating } \\
\text { Composition }\end{array}$ & $\begin{array}{c}\text { Solids } \\
\text { Percent } \\
\text { of PVOH } \\
\text { Stock } \\
\text { (wt.\%) }\end{array}$ & $\begin{array}{c}\text { Mass } \\
\text { of } \\
\text { PVOH } \\
\text { stock } \\
\text { (g) }\end{array}$ & $\begin{array}{c}\text { Mass } \\
\text { of } \\
\text { VTMS } \\
\text { (g) }\end{array}$ & $\begin{array}{c}\text { Mass } \\
\text { of DI } \\
\text { Water } \\
\text { (g) }\end{array}$ & $\begin{array}{c}\text { Solids } \\
\text { Percent } \\
\text { of PVOH/ } \\
\text { VTMS } \\
\text { Blend } \\
\text { (wt.\%) }\end{array}$ & $\begin{array}{c}\text { Viscosity } \\
\text { at 24 } \\
\text { hours } \\
\text { (mPa.s) }\end{array}$ \\
\hline $\begin{array}{l}\text { 90 wt.\% } \\
\text { PVOH/ 10 } \\
\text { wt.\% PVOH }\end{array}$ & 16.82 & 2099.1 & 93 & 41.7 & 16.11 & 160 \\
\hline
\end{tabular}

With this formulation, eight overall variables were tested in a total of four printing runs on a Mark Andy 2200 Series flexographic printing press (Figure 12). The two sleeves allowed for subsequent layers of coating to be deposited, with about a 1 minute delay between layer additions. Two sets of anilox rolls were paired to replicate the double-layer procedure used when preparing films on the QD Proofer. The first set of anilox rolls was made up by a full width 600 CPI 2.48 BCM roll, followed by a half width 6002.24 roll. The second set of anilox rolls included a full width 3606.95 roll, followed by a half width 3606.53 roll. Both of these anilox roll pairs were also used in combination with two variations of tint sleeves, the M503 sleeve and the M541 sleeve. Films prepared using the Mark Andy press had two halves to them, one half with a single layer coating from the first anilox roll (i.e. 600 2.48), and the second half with a doublelayer coating deposited by the set of anilox rolls (i.e. 6002.24 and 6002.48 ). 

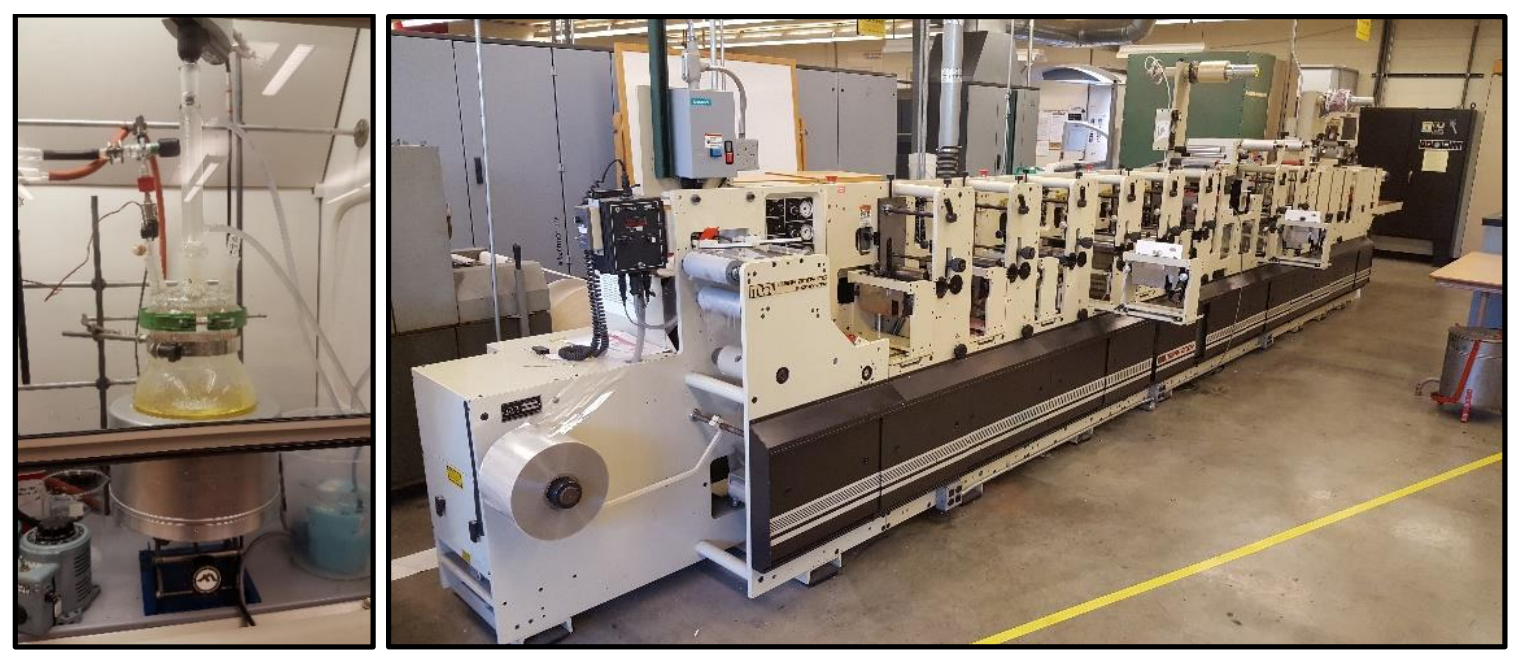

Figure 12. Images of the up scaled, round bottom reaction setup (left) and Mark Andy flexographic press (right)

An immediate challenge faced while running the press trial was that the coronatreated 12 micron thick PET, used previously on the QD Proofer, didn't run smoothly through the press. The Mark Andy flexographic press was designed use thicker substrates, and as a result, significant creasing was observed in the resultant films despite attempts to adjust the printing speed and the line tension. In the end, the printing speed was reduced down to 75 feet/minute to minimize damage to the substrate, and allow for adjustments as the film weaved through the press. The printing press had six ovens across its entire length, four after the first sleeve and two after the second sleeve that were heated to $53^{\circ} \mathrm{C}$. At the speed of 75 feet/minute, the dwell time of the films within each oven was approximately half a second.

A 2 mil PET substrate was obtained and run through the same set of variable conditions as the 12 micron PET to provide undamaged films for OTR testing. This 2 mil PET had no difficulties running through the Mark Andy press at $75 \mathrm{feet} / \mathrm{minute}$. The films prepared from this second press trial were significantly better than those previously 
produced on the 12 micron PET substrate. The 90/10 PVOH/VTMS coating kept the cylinder rolls wet throughout the press trials, and didn't seem to deteriorate after several hours of use. About $500 \mathrm{~mL}$ of PVOH/VTMS coating was used in each sleeve tray accounting for $1 \mathrm{~L}$ total volume. Approximately $700 \mathrm{~mL}$ was recovered after the coating had been transferred back to its bottle.

Typical analysis methods were conducted on these 2 mil PET films before submitting them for OTR testing. Unfortunately, the thickness determination previously used with the coated 12 micron PET films didn't produce consistent thicknesses for the coated 2 mil PET films. The thicknesses of the eight coating layers was undetermined since no other method was available. Polarized optical microscopy was used to capture the images shown in Figure 13. The films prepared with the 2 BCM anilox rolls displayed the usual ellipsoidal shapes in the single layer variations, while neither of the films prepared with the $6 \mathrm{BCM}$ anilox rollers appeared to exhibit these distinct regions. The coating consistency seemed to be better using the $6 \mathrm{BCM}$ rolls due to a thicker coating. There appear to be holes and defects present in the $2 \mathrm{BCM}$ roller films that are highlighted by blue circles in Figure 13 . 


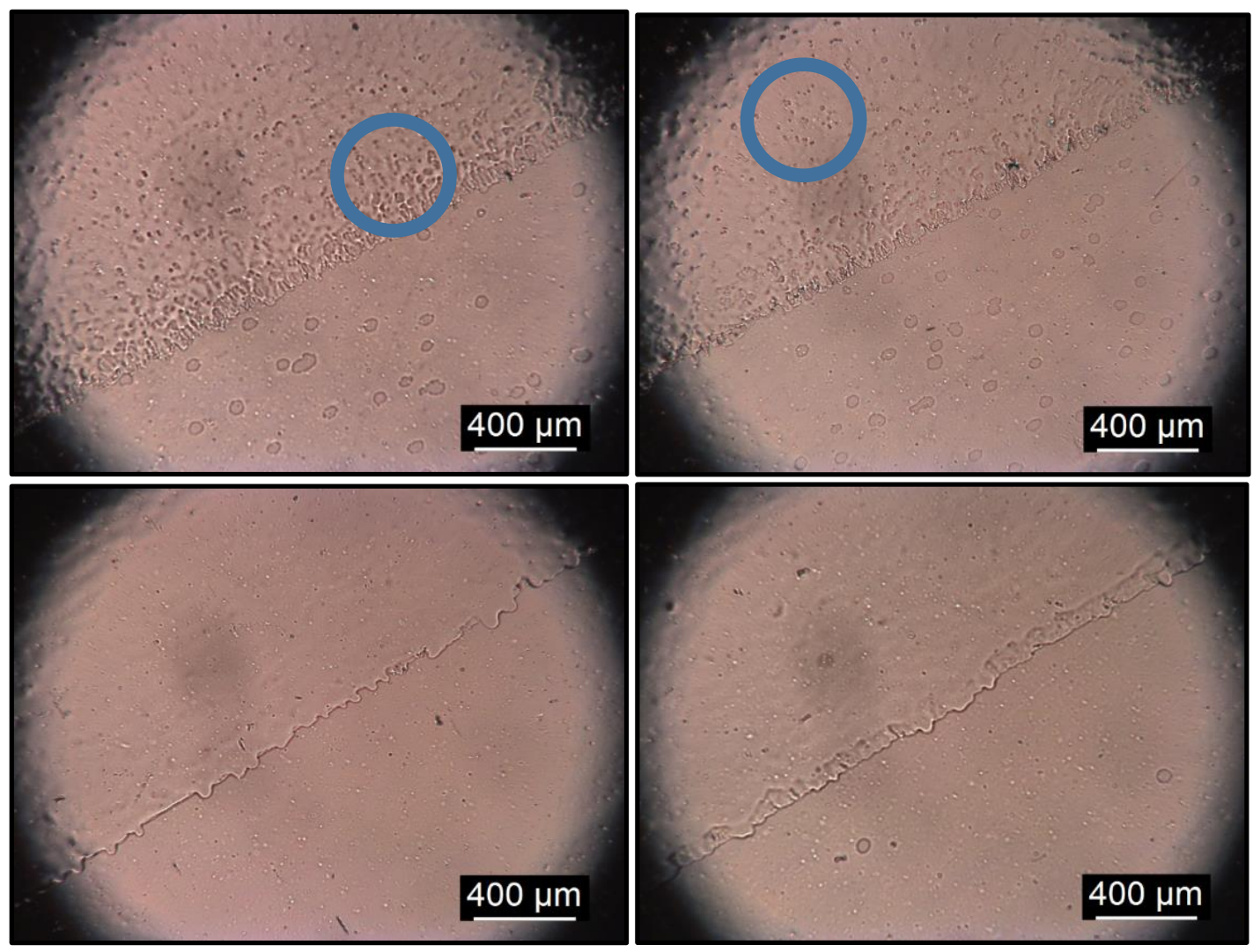

Figure 13. POM images of M504 2 BCM (top left), M541 2 BCM (top right), M504 6 BCM (bottom left), and M541 6 BCM (bottom right) films at 5x magnification with double-layers above and single-layers below each diagonal line

The 2 mil PET substrate produced an OTR of $48.38 \mathrm{cc} \mathrm{m}^{-2}$ day $^{-1}$ at $0 \% \mathrm{RH}$, showing how the increase in thickness reduces the OTR by over $50 \%$ compared to the 12 micron PET substrate. The 2 BCM single layer film was tested first 24 hours after being printed, yielding an OTR of $19.75 \mathrm{cc} \mathrm{m}^{-2} \mathrm{day}^{-1}(59.2 \%$ reduction). This OTR was significantly higher than films prepared with the QD Proofer on 12 micron PET, leading to doubts about whether these films were fully cured. This may be true, due to the short oven dwell times that the films experience while running through the press. No additional curing treatments were done with these films after being prepared on the Mark Andy flexographic printing press. 
To ensure complete curing of these 90/10 PVOH/VTMS films, unrolled film samples were placed in the oven at $70^{\circ} \mathrm{C}$ for an hour. This force curing took place several weeks after the press trial. Visual inspection and POM imaging didn't highlight any noticeable differences in the force cured films, when compared to the original films. The oxygen transmission rates for these force cured 6 BCM films are given in Table 26.

Table 26. Oxygen transmission rates and thicknesses obtained for the 2 mil PET press trial films using the 90/10 PVOH/VTMS blend

\begin{tabular}{|l|c|c|c|}
\hline \multicolumn{1}{|c|}{ Coating Composition } & $\begin{array}{c}\text { Substrate } \\
\text { Thickness } \\
(\boldsymbol{\mu m})\end{array}$ & $\begin{array}{c}\text { OTR at 0\% RH } \\
\left(\mathbf{c c ~ m}^{-\mathbf{2}} \mathbf{d a y}^{\mathbf{- 1})}\right.\end{array}$ & $\begin{array}{c}\text { OTR at 90\% RH } \\
\left(\mathbf{c c ~ m}^{-\mathbf{2}} \mathbf{d a y}^{\mathbf{1}}\right)\end{array}$ \\
\hline 2 mil Press PET & 50.8 & $48.38 \pm 1.43$ & \\
\hline $\begin{array}{l}2 \text { mil PET- M541 2 BCM } \\
\text { Single Layer }\end{array}$ & N/A & $19.75 \pm 1.23$ & \\
\hline $\begin{array}{l}2 \text { mil PET- M541 6 BCM } \\
\text { Double-Layer (Force- } \\
\text { Cured) }\end{array}$ & N/A & $2.25 \pm 1.48$ & \\
\hline $\begin{array}{l}2 \text { mil PET- M541 6 BCM } \\
\text { Single Layer (Force- } \\
\text { Cured) }\end{array}$ & N/A & $6.60 \pm 0.84$ & $6.75 \pm 0.64$ \\
\hline
\end{tabular}

The two post-cure films prepared the $6 \mathrm{BCM}$ anilox rolls display OTRs of $6.60 \mathrm{cc}$ $\mathrm{m}^{-2} \operatorname{day}^{-1}$ (86.4\% reduction) for the single layer and $6.60 \mathrm{cc} \mathrm{m}^{-2}$ day $^{-1}$ (95.4\% reduction) for the double-layer, that are representative of high oxygen barrier performance. However, given the thickness of the 2 mil PET substrate, the OTRs associated with properly cured films of the 90/10 PVOH/VTMS blend should be expected to be well below $1 \mathrm{cc} \mathrm{m}^{-2}$ day $^{-1}$. Once more optimal methods of curing these films are developed, the PVOH/VTMS coatings developed during this project shows great promise in providing excellent oxygen barrier performance that is maintained between low and high humidity. 


\section{CONCLUSIONS}

A strong oxygen barrier coating that promotes near full retention of oxygen barrier performance at high humidity was achieved during this project. The resin structure of this coating is created following a condensation reaction mechanism between PVOH chains and VTMS molecules; resembling the network created in common sol-gel systems. The properties of the 90/10 PVOH/VTMS blend can be varied by controlling the solids content and viscosity, which have a direct impact on the thickness and consistency of the dry coating. As long as no holes or defects were present in the dry coated film, oxygen transmission rates of $1-2 \mathrm{cc} \mathrm{m}^{-2}$ day $^{-1}$ were demonstrated using the 90/10 PVOH/VTMS composition. The thickness of the dry coating was typically $1-2$ microns. The blend prepared with citric acid as a catalyst appears to have slightly improved oxygen barrier performance at both $0 \%$ and $90 \%$ RH compared to those prepared with hydrochloric acid, ascribed to additional crosslinking within the PVOH/VTMS resin structure. The trial press, run on the Mark Andy flexographic printing press, was limited to a low deposition rate of $75 \mathrm{ft} / \mathrm{min}$ due to incompatibility with the 12 micron PET substrate. Films prepared using an alternate 2 mil PET substrate were ideal, with the 90/10 PVOH/VTMS blend demonstrating consistent and durable use throughout the trial run. While adjustments of certain aspects of large scale film preparation and the curing process must be made to optimize the coating performance, the development of this oxygen barrier coating achieved during the project provides a substantial basis for a novel oxygen barrier coating that could be used for commercial food packaging applications. 


\section{REFERENCES}

1. Kummu, M.; de Moel, H.; Porkka, M.; Siebert.; Varis, O.; Ward, P.J. Lost food, wasted resources: Global food supply chain losses and their impacts on freshwater, cropland, and fertiliser use. Science of the Total Environment. 2012, $438,477-489$

2. Kantor, L.; Lipton, K.; Manchester, A.; Oliveira, V. Estimating and Addressing America's Food Losses. Food Loss. 1997, 1-11

3. Gustavsson, J.; Cederberg, C.; Sonesson, U.; van Otterdijk, R.; Meybeck, A. Global Food Losses and Food Waste. Food and Agriculture Organization of the United Nations. 2011, 1-23

4. Coles, R.; McDowell, D.; Kirwan, M. Food Packaging Technology. Blackwell Publishing Ltd. 2003, 1-208

5. Amberg-Schwab, S.; Weber, U.; Burger, A.; Nique, S.; Xalter, R. Development of Passive and Active Barrier Coatings on the Basis of Inorganic-Organic Polymers. Monatshefte fur Chemie. 2006, 137, 657-666

6. Esterbauer, H. Cytotoxicity and genotoxicity of lipid-oxidation products. American Society for Clinical Nutrition. 1993, 57, 779-786

7. Bekhit, A.D.; Faustman, C. Metmyoglobin reducing activity. Meat Science. 2005, 71, 407-439

8. Bonilla, J.; Atares, L.; Vargas, M.; Chiralt, A. Edible films and coatings to prevent the detrimental effect of oxygen on food quality: Possibilities and limitations. Journal of Food Engineering. 2012, 110, 208-213

9. Jameison, E.H.; Windle, A.H. Structure and oxygen-barrier properties of metallized polymer film. Journal of Materials Science. 1983, 18, 64-80

10. Schroeder, G. Multi-layer barrier film. United States Patent. 1981, 1-5

11. Beckett, D. Formation of Packaging Material. United States Patent. 1983, 1-4

12. Lutzmann, H.; Miller, G. Enhanced Barrier Compositions from Polyvinyl Alcohol. United States Patent. 1986, 1-3

13. Shah, G. Oxygen Barrier Oriented Srink Film. United States Patent. 1988, 1-8 
14. Schmukler, S.; Shida, M.; Machonis, J. Polyvinyl Alcohol Alloys and Method of Making the Same. United States Patent. 1986, 1-12

15. Hirvikorpi, T.; Vaha-Nissi, M.; Nikkola, J.; Harlin, A.; Karppinen, M. Thin A12O3 barrier coatings onto temperature-sensitive packaging materials by atomic layer deposition. Surface \& Coatings Technology. 2011, 205, 5088-5092

16. Logothetidis, S.; Laskarakis, A.; Georgiou, D.; Amberg-Schwab, S.; Weber, U.; Noller, K.; Schmidt, M.; Kucukpinar, E.; Lohwasser, W. Ultra high barrier materials for encapsulation of flexible organic electronics. Physics and Astronomy Classification Scheme. 2010, 78, 1-18

17. Leterrier, Y.; Singh, B.; Bouchet, J.; Manson, J.; Rochat, G.; Fayet, P. Supertough UV-curable silane/silica gas barrier coatings on polymers. Surface \& Coatings Technology. 2009, 203, 3398-3404

18. Prager, L.; Dierdorf, A.; Liebe, H.; Naumov, S.; Stojanovic, S.; Heller, R.; Wennrich, L.; Buchmeiser, M. Conversion of Perhydropolysilazane into a SiOx Network Triggered by Vacuum Ultraviolet Irradiation: Access to Flexible, Transparent Barrier Coatings. Chemistry European Journal. 2007, 13, 8522-8529

19. Mannle, F.; Tofteberg, T.; Skaugen, M.; Bu, H.; Peters, T.; Dietzel, P.; Pilz, M.; Polymer nanocomposite coatings based on polyhedral oligosilsesquioxanes: route for industrial manufacturing and barrier properties. Journal of Nanoparticle Research. 2011, 13, 4691-4701

20. Dou, Y.; Zhou, A.; Pan, T.; Han, J.; Wei, M.; Evans, D.; Duan, X. Humidity-triggered self-healing films with excellent oxygen barrier performance. Chemical Communications. 2014, 1-3

21. Moller, M.; Kunz, D.; Lunkenbein, T.; Sommer, S.; Nennemann, A.; Breu, J. UVCured, Flexible, and Transparent Nanocomposite Coating with Remarkable Oxygen Barrier. Advanced Materials. 2012, 24, 2142-2147

22. Priolo, M.; Gamboa, D.; Grunlan, J. Transparent Clay-Polymer Nano Brick Wall Assemblies with Tailorable Oxygen Barrier. Applied Materials \& Interfaces. 2010, 2, 1, 312-320

23. Leterrier, Y. Durability of nanosized oxygen-barrier coatings on polymers. Progress in Material Science. 2003, 14, 1-5

24. Minelli, M.; De Angelis, M.; Doghieri, F.; Rocchetti, M.; Montenero, A. Barrier Properties of Organic-Inorganic Hybrid Coatings Based on Polyvinyl Alcohol With Improved Water Resistance. Polymer Engineering and Science. 2010, 144153 
25. ASTM Standard D3985-05, 'Standard Test Method for Oxygen Gas Transmission Rate Through Plastic Film and Sheeting Using a Coulometric Sensor.' ASTM International. 2010

26. Chatnam, H. Oxygen diffusion barrier properties of transparent oxide coatings on polymeric substrates. Surface \& Coatings Technology. 1996, 78, 1-9

27. Robertson, G. Food Packaging Principles and Practice. Taylor \& Francis Group. 2013, 1-16

28. Marsh, K.; Bugusu, B. Food Packaging - Roles, Materials, and Environmental Issues. Institute of Food Technologies. 2007, 72, 3, R39-55

29. Mokwena, K.; Tang, J. Ethylene Vinyl Alcohol: A Review of Barrier Properties for Packaging Shelf Stable Foods. Critical Reviews in Food Science and Nutrition. 2012, 52, 640-650

30. Miller, K.S.; Krochta, J.M.; Oxygen and Aroma Barrier Properties of Edible Films: A Review. Trends in Food Science \& Technology. 1997, 8, 228-237

31. Peppas, N.; Hansen, P. Crystallization Kinetics of Poly(vinyl alcohol). Journal of Applied Polymer Science. 2003, 27, 1-11.

32. DeMerlis, C.; Schoneker, D.; Review of the oral toxicity of polyvinyl alcohol (PVA). Food and Chemical Toxicology. 2003, 41, 319-326

33. Fabra, M.; Lopez-Rubio, A.; Lagaron, J. On the use of different hydrocolloids as electrospun adhesive interlayers to enhance the barrier properties of polyhydroxyalkanoates of interest in fully renewable food packaging concepts. Food Hydrocolloids, 2014, 39, 77-84

34. Wen, J.; Wilkes, G. Organic/Inorganic Hybrid Network Materials by the Sol-Gel Approach. Chemical Materials, 1996, 8, 1667-1681

35. Farris, S.; Unalan, I.; Introzzi, L., Fuentes-Alventosa, J.; Cozzolino, C. PullulanBased Films and Coatings for Food Packaging: Present Applications, Emerging Opportunities, and Future Challenges. Applied Polymer Science. 2014, 40539, 1 12

36. Fox, R.; Zhang, H.; Barger, M.; Han, C.; Paquette, M. Natural and synthetic clayfilled coatings for insulation barrier applications. Journal of Coating Technology and Research. 2016, 13, 1, 181-189

37. Nyflott, A. The influence of clay orientation and crystallinity on oxygen permeation in dispersion barrier coatings. Applied Clay Science. 2016, 126, 17-24 
38. Duncan, T. Application of nanotechnology in food packaging and food safety: Barrier materials, antimicrobials and sensors. Journal of Colloid and Interfacial Science. 2011, 1-24

39. Grunlan, J.; Grigorian, A.; Hamilton, C.; Mehrabi, A. Effect of Clay Concentration on the Oxygen Permeability and Optical Properties of a Modified Poly(vinyl alcohol). Journal of Applied Polymer Science. 2004, 93, 1102-1109

40. Borriello, C.; De Maria, A.; Jovic, N.; Montone, A.; Schwarz, M.; Vittori, J.; Antisari, M.; Mechanochemical exfoliation of graphite and its polyvinyl alcohol nanocomposites with enhanced barrier properties. Materials and Manufacturing Processes. 2009, 24, 1053-1057

41. Paralikar, S.; Simonsen, J.; Lombardi, J.; Poly(vinyl alcohol)/cellulose nanocrystal barrier membranes. Journal of Membrane Science. 2008, 320, 248-258

42. Azeredo, H. Nanocomposites for food packaging applications. Food Research International. 2009, 42, 1240-1253

43. Riegel, B.; Blittersdorf, S.; Kiefer, W.; Hofacker, S.; Muller, M.; Schottner, G. Kinetic Investigation of Hydrolysis and Condensation of the Glycidoxypropyl Trimethoxysilane/Aminopropyltriethoxysilane System by Means of FT-Raman Spectroscopy I. Journal of Non-Crystalline Solids. 1998, 226, 76-84

44. Zhang, X.; Wu, W.; Xia, X.; Zhang, Z.; Yu, R. Toughening of cycloaliphatic epoxy resin by nanosize silicon dioxide. Materials Letters. 2006, 60, 3319-3323

45. Bae, J.; Lee, J.; Park, C.; Kwon, O.; Lee, C. Fabrication of photo-crosslinkable polymer/silica sol-gel hybrid thin films as versatile barrier films. Journal of Industrial and Engineering Chemistry. 2016, 38, 61-66

46. Lim, M.; Kim, D.; Seo, J.; Han, H. Preparation and Properties of Poly(vinyl alcohol)/Vinyltrimethoxysilane (PVA/VTMS) Hybrid Films with Enhanced Thermal Stability and Oxygen Barrier Properties. Macromolecular Research. 2014, 22, 10, 1096-1103

47. Moller, H.; Grelier, S.; Pardon, P.; Coma, V. Antimicrobial and Physicochemical Properties of Chitosan-HPMC-Based Films. Journal of Agricultural Food Chemistry. 2004, 52, 6585-6591 